\title{
Topological interpretations of provability logic
}

\author{
Lev Beklemishev* David Gabelaia ${ }^{\dagger}$
}

May 2, 2018

\begin{abstract}
Provability logic concerns the study of modality $\square$ as provability in formal systems such as Peano arithmetic. Natural, albeit quite surprising, topological interpretation of provability logic has been found in the 1970's by Harold Simmons and Leo Esakia. They have observed that the dual $\diamond$ modality, corresponding to consistency in the context of formal arithmetic, has all the basic properties of the topological derivative operator acting on a scattered space. The topic has become a long-term project for the Georgian school of logic led by Esakia, with occasional contributions from elsewhere.

More recently, a new impetus came from the study of polymodal provability logic GLP that was known to be Kripke incomplete and, in general, to have a more complicated behavior than its unimodal counterpart. Topological semantics provided a better alternative to Kripke models in the sense that GLP was shown to be topologically complete. At the same time, new fascinating connections with set theory and large cardinals have emerged.

We give a survey of the results on topological semantics of provability logic starting from first contributions by Esakia. However, a special emphasis is put on the recent work on topological models of polymodal provability logic. We also included a few results that have not been published so far, most notably the results of Section 6 (due the second author) and Sections 10, 11 (due to the first author).
\end{abstract}

\section{Provability algebras and logics}

Provability logics and algebras emerge from, respectively, a modal logical and an algebraic point of view on the proof-theoretic phenomena around Gödel's incompleteness theorems. These theorems are usually perceived as putting fundamental restrictions on what can be formally proved in a given axiomatic system (satisfying modest natural requirements). For the sake of a discussion, we call a formal theory $T$ gödelian if

- $T$ is a first order theory in which the natural numbers along with the operations + and $\cdot$ are interpretable;

\footnotetext{
*V.A. Steklov Mathematical Institute, RAS; Moscow M.V. Lomonosov State University; National Research University Higher School of Economics; email: bekl@mi.ras.ru

${ }^{\dagger}$ TSU Razmadze Institute of Mathematics, Tbilisi; email: gabelaia@gmail.com
} 
- $T$ proves some basic properties of these operations and a modicum of induction (it is sufficient to assume that $T$ contains the Elementary Arithmetic EA);

- $T$ has a recursively enumerable set of axioms.

The Second Incompleteness Theorem of Kurt Gödel (G2) states that a gödelian theory $T$ cannot prove its own consistency provided it is indeed consistent. More accurately, for any r.e. presentation of such a theory $T$, Gödel has shown how to write down an arithmetical formula $\operatorname{Prov}_{T}(x)$ expressing that $x$ is (a natural number coding) a formula provable in $T$. Then the statement $\operatorname{Con}(T):=\neg \operatorname{Prov}_{T}(\ulcorner\perp\urcorner)$ naturally expresses that the theory $T$ is consistent. G2 states that $T \nvdash \operatorname{Con}(T)$ provided $T$ is consistent.

Provability logic emerged from the question what properties of formal provability $\operatorname{Prov}_{T}$ can be verified in $T$, even if the consistency of $T$ cannot. Several such properties have been stated by Gödel himself [30]. Hilbert and Bernays and then Löb 38 stated them in the form of conditions any adequate formalization of a provability predicate in $T$ must satisfy. After Gödel's and Löb's work it was clear that the formal provability predicate calls for a treatment as a modality. It led to the formulation of the Gödel-Löb provability logic GL and eventually to the celebrated arithmetical completeness theorem due to Robert Solovay.

Independently, Macintyre and Simmons [39] and Magari [40] took a very natural algebraic perspective on the phenomenon of formal provability which led to the concept of diagonalizable algebra. Such algebras are now more commonly called Magari algebras. This point of view will be more convenient for our present purposes.

Recall that the Lindenbaum-Tarski algebra of a theory $T$ is the set of all $T$ sentences Sent $_{T}$ modulo provable equivalence in $T$, that is, the structure $\mathcal{L}_{T}=$ $\operatorname{Sent}_{T} / \sim_{T}$ where, for all $\varphi, \psi \in \operatorname{Sent}_{T}$,

$$
\varphi \sim_{T} \psi \Longleftrightarrow T \vdash(\varphi \leftrightarrow \psi) .
$$

Since we assume $T$ to be based on classical propositional logic, $\mathcal{L}_{T}$ is a boolean algebra with operations $\wedge, \vee, \neg$. Constants $\perp$ and $T$ are identified with the sets of refutable and of provable sentences of $T$, respectively. The standard ordering on $\mathcal{L}_{T}$ is defined by

$$
[\varphi] \leq[\psi] \Longleftrightarrow T \vdash \varphi \rightarrow \psi \Longleftrightarrow[\varphi \wedge \psi]=[\varphi],
$$

where $[\varphi]$ denotes the equivalence class of $\varphi$.

It is well-known that for consistent gödelian $T$ all such algebras are isomorphic to the unique countable atomless boolean algebra. (This is a consequence of a strengthening of Gödel's First Incompleteness Theorem due to Rosser.) We obtain more interesting algebras by enriching the structure of the boolean algebra $\mathcal{L}_{T}$ by additional operation(s).

Gödel's consistency formula induces a unary operator $\diamond_{T}$ acting on $\mathcal{L}_{T}$ :

$$
\diamond_{T}:[\varphi] \longmapsto[\operatorname{Con}(T+\varphi)]
$$

The sentence Con $(T+\varphi)$ expressing the consistency of $T$ extended by $\varphi$ can be defined as $\neg \operatorname{Prov}_{T}(\ulcorner\neg \varphi\urcorner)$. The dual operator is $\square_{T}:[\varphi] \longmapsto\left[\operatorname{Prov}_{T}(\ulcorner\varphi\urcorner)\right]$, thus $\square_{T} x=\neg \diamond_{T} \neg x$, for all $x \in \mathcal{L}_{T}$. 
Bernays-Löb derivability conditions ensure that $\diamond_{T}$ is correctly defined on the equivalence classes of the Lindenbaum-Tarski algebra of $T$. Moreover, it satisfies the following identities (where we write $\diamond_{T}$ simply as $\diamond$ and the variables range over arbitrary elements of $\left.\mathcal{L}_{T}\right)$ :

M1. $\diamond \perp=\perp ; \quad \diamond(x \vee y)=\diamond x \vee \diamond y$;

M2. $\diamond x=\diamond(x \wedge \neg \diamond x)$.

Notice that Axiom M2 is a formalization of G2 stated for the theory $T^{\prime}=T+\varphi$, where $[\varphi]=x$. In fact, the left hand side states that $T^{\prime}$ is consistent, whereas the right hand side states that $T^{\prime}+\neg \operatorname{Con}\left(T^{\prime}\right)$ is consistent, that is, $T^{\prime} \nvdash \operatorname{Con}\left(T^{\prime}\right)$. The dual form of Axiom M2,

$$
\square(\square x \rightarrow x)=\square x,
$$

expresses the familiar Löb's theorem.

Notice also that M1 induces $\diamond$ to be monotone: if $x \leq y$ then $\diamond x \leq \diamond y$.

Boolean algebras with operators $\mathcal{M}=(M, \diamond)$ satisfying the above identities are called Magari algebras. Thus, the main example of a Magari algebra is the structure $\left(\mathcal{L}_{T}, \diamond_{T}\right)$, for any consistent gödelian theory $T$, which is also called the provability algebra of $T$.

The transitivity inequality $\diamond \diamond x \leq \diamond x$ is often postulated as an additional axiom of Magari algebras, however it follows from M1 and M2.

Proposition 1.1. In any Magari algebra $\mathcal{M}$, there holds $\diamond \diamond x \leq \diamond x$, for all $x \in M$.

Proof. Given any $x \in M$ consider $y:=x \vee \diamond x$. On the one hand, we have

$$
\diamond \diamond x \leq(\diamond x \vee \diamond \diamond x)=\diamond y
$$

On the other hand, since $\diamond x \wedge \neg \diamond y=\perp$ we obtain

$$
\diamond y \leq \diamond(y \wedge \neg \diamond y) \leq \diamond((x \vee \diamond x) \wedge \neg \diamond y) \leq \diamond(x \wedge \neg \diamond y) \vee \diamond \perp \leq \diamond x .
$$

Hence, $\diamond \diamond x \leq \diamond x$.

In general, we call an identity of an algebraic structure $\mathcal{M}$ a formula of the form $t(\vec{x})=u(\vec{x})$, where $t, u$ are terms, such that $\mathcal{M} \vDash \forall \vec{x}(t(\vec{x})=u(\vec{x}))$. Identities of Maragi algebras can be described in terms of modal logic as follows. Any term (built from the variables using boolean operations and $\diamond$ ) is naturally identified with a formula in the language of propositional logic with a new unary connective $\diamond$. If $\varphi(\vec{x})$ is such a formula and $\mathcal{M}$ a Magari algebra, we write $\mathcal{M} \vDash \varphi$ iff $\forall \vec{x}\left(t_{\varphi}(\vec{x})=\top\right)$ is valid in $\mathcal{M}$, where $t_{\varphi}$ is the term corresponding to $\varphi$. Since any identity in Magari algebras can be equivalently written in the form $t=T$, for some term $t$, the axiomatization of identities of $\mathcal{M}$ amounts to axiomatizing modal formulas valid in $\mathcal{M}$. The $\operatorname{logic}$ of $\mathcal{M}, \log (\mathcal{M})$, is the set of all modal formulas valid in $\mathcal{M}$, that is,

$$
\log (\mathcal{M}):=\{\varphi: \mathcal{M} \vDash \varphi\},
$$

and the logic of a class of modal algebras is defined similarly.

One of the main parameters of a Magari algebra $\mathcal{M}$ is its characteristic $\operatorname{ch}(\mathcal{M})$ which is defined as follows. We let

$$
\operatorname{ch}(\mathcal{M}):=\min \left\{k \in \omega: \diamond^{k} \top=\perp\right\}
$$


and $\operatorname{ch}(\mathcal{M}):=\infty$, if no such $k$ exists. If $T$ is arithmetically sound, that is, if the arithmetical consequences of $T$ are valid in the standard model, then $\operatorname{ch}\left(\mathcal{L}_{T}\right)=\infty$. Theories of finite characteristics are, in a sense, close to being inconsistent and can be considered a pathology.

Robert Solovay in his fundamental paper [46] proved that any identity valid in the structure $\left(\mathcal{L}_{T}, \diamond_{T}\right)$ follows from the boolean identities together with M1-M2, provided $T$ is arithmetically sound. This has been generalized by Albert Visser [48] to arbitrary theories of infinite characteristic. Put algebraically, this becomes the following statement.

Theorem 1.2. Suppose $\operatorname{ch}\left(\mathcal{L}_{T}, \diamond_{T}\right)=\infty$. An identity holds in $\left(\mathcal{L}_{T}, \diamond_{T}\right)$ iff it holds in all Magari algebras.

Apart from the equational characterization by M1-M2 above, the identities of Magari algebras can be axiomatized modal-logically. In fact, the logic of all Magari algebras, and by Solovay theorem the $\operatorname{logic} \log \left(\mathcal{L}_{T}, \diamond_{T}\right)$ of the Magari algebra of $T$, for any fixed theory $T$ of infinite characteristic, coincides with the familiar Gödel-Löb logic GL. By abuse of language we will often identify GL with the set of identities of Magari algebras.

A Hilbert-style axiomatization of $\mathbf{G L}$ is usually given in the modal language where $\square$ rather than $\diamond$ is taken as basic and the latter is treated as an abbreviation for $\neg \square \neg$. The axioms and inference rules of GL are as follows.

\section{Axiom schemata:}

L1. All instances of propositional tautologies;

L2. $\square(\varphi \rightarrow \psi) \rightarrow(\square \varphi \rightarrow \square \psi)$;

L3. $\square(\square \varphi \rightarrow \varphi) \rightarrow \square \varphi$.

Rules: $\varphi, \varphi \rightarrow \psi / \psi$ (modus ponens), $\varphi / \square \varphi$ (necessitation).

By a well-known result of Segerberg, GL is sound and complete w.r.t. the class of all transitive and upwards well-founded Kripke frames. In fact, it is sufficient to restrict the attention to frames that are finite irreflexive trees. Thus, summarizing various characterizations above, we have

Theorem 1.3. For any modal formula $\varphi$, the following statements are equivalent:

(i) $\mathbf{G L} \vdash \varphi$;

(ii) $\varphi$ is valid in all Magari algebras;

(iii) $\left(\mathcal{L}_{T}, \diamond_{T}\right) \vDash \varphi$, for any $T$ of infinite characteristic;

(iv) $\varphi$ is valid in all finite irreflexive tree-like Kripke frames.

\section{Topological interpretation}

Having defined the notion of Magari algebra, the first question one can ask is whether there are any other natural examples of such algebras apart from the provability algebras of the form $\left(\mathcal{L}_{T}, \diamond_{T}\right)$. The fact that such algebras naturally emerge from scattered topological spaces was discovered independently by Harold 
Simmons [44] and Leo Esakia [27, 1] We now infer this semantics from rather general considerations.

It is a standard idea in logic, going back at least as early as to Leibnitz, to interpret propositions as subsets of a given nonempty set $X$. Then, logical connectives are naturally translated as the familiar boolean operations on sets. From this point of view, $\diamond$ should correspond to an operator acting on the subsets of $X$, so we come to the following mathematical question.

Let $X$ be a nonempty set, $\mathcal{P}(X)$ the boolean algebra of subsets of $X$. Consider any operator $\delta: \mathcal{P}(X) \rightarrow \mathcal{P}(X)$ and the structure $(\mathcal{P}(X), \delta)$. Can $(\mathcal{P}(X), \delta)$ be a Magari algebra and, if yes, when? Simmons and Esakia, in some sense, found a canonical answer to this question.

Let $(X, \tau)$ be a topological space, where $\tau$ denotes the set of open subsets of $X$, and let $A \subseteq X$. Topological derivative $d_{\tau}(A)$ of $A$ is the set of limit points of $A$ :

$$
x \in d_{\tau}(A) \Longleftrightarrow \forall U \in \tau(x \in U \Rightarrow \exists y \neq x(y \in U \cap A)) .
$$

Notice that $c_{\tau}(A):=A \cup d_{\tau}(A)$ is the closure of $A$ and $i_{\tau}(A):=A \backslash d_{\tau}(A)$ is the set of isolated points of $A$.

The classical notion of a scattered topological space is due to Georg Cantor. $(X, \tau)$ is called scattered if every nonempty subspace $A \subseteq X$ has an isolated point.

Theorem 2.1 (Simmons, Esakia). The following statements are equivalent:

(i) $(X, \tau)$ is scattered;

(ii) For all $A \subseteq X, d_{\tau}(A)=d_{\tau}\left(A \backslash d_{\tau}(A)\right)$;

(iii) $\left(\mathcal{P}(X), d_{\tau}\right)$ is a Magari algebra.

Notice that (ii) means that each point of $A$ is a limit point of its isolated points. Algebras of the form $\left(\mathcal{P}(X), d_{\tau}\right)$ associated with a topological space $(X, \tau)$ will be called derivative algebras. Thus, this theorem states that the derivative algebra of $(X, \tau)$ is Magari iff $(X, \tau)$ is scattered.

Proof. First, we notice that Statement (iii) is just a reformulation of (ii). In fact, the identities $d_{\tau} \varnothing=\varnothing$ and $d_{\tau}(A \cup B)=d_{\tau}(A) \cup d_{\tau}(B)$ are obviously valid in all topological spaces. We show the equivalence of (i) and (ii).

Suppose $(X, \tau)$ is scattered, $A \subseteq X$ and $x \in d_{\tau}(A)$. Consider any open neighborhood $U$ of $x$. Since $U \cap A \backslash\{x\}$ is nonempty, it has an isolated point $y \neq x$. Since $U$ is open, $y$ is an isolated point of $A$, that is, $y \in A \backslash d_{\tau}(A)$. Hence, $x \in d_{\tau}\left(A \backslash d_{\tau}(A)\right)$. The inclusion $d_{\tau}\left(A \backslash d_{\tau}(A)\right) \subseteq d_{\tau}(A)$ follows from the monotonicity of $d_{\tau}$. Therefore Statement (ii) holds.

Suppose that (ii) holds and let $A \subseteq X$ be nonempty. We show that $A$ has an isolated point. If $d_{\tau} A$ is empty, we are done. Otherwise, take any $x \in d_{\tau} A$. Since $x$ is a limit of isolated points of $A$, there must be at least one such point.

We notice that the transitivity principle $d_{\tau} d_{\tau} A \subseteq d_{\tau} A$ topologically means that the set $d_{\tau} A$, for any $A \subseteq X$, is closed. We recall the following standard equivalent characterization an easy proof of which we shall omit.

\footnotetext{
${ }^{1}$ The paper by Simmons was published in the pre-Solovay era of provability logic and remained largely ignored. Esakia apparently independently discovered the interpretation when he was already familiar with the works of Magari and Solovay.
} 
Proposition 2.2. For any topological space $(X, \tau)$, the following statements are equivalent:

(i) Every $x \in X$ is an intersection of an open and a closed set;

(ii) For each $A \subseteq X$, the set $d_{\tau} A$ is closed.

Topological spaces satisfying either of these conditions are called $T_{d}$-spaces. Condition (i) shows that $T_{d}$ is a weak separation property located between $T_{0}$ and $T_{1}$. Thus, Proposition 1.1 yields the following corollary.

Corollary 2.3. All scattered spaces are $T_{d}$.

We have seen in Theorem 2.1 that each scattered space equipped with a topological derivative operator is a Magari algebra. The following result shows that any Magari algebra on $\mathcal{P}(X)$ can be described in this way.

Theorem 2.4. If $(\mathcal{P}(X), \delta)$ is a Magari algebra, then $X$ bears a unique topology $\tau$ for which $\delta=d_{\tau}$. Moreover, $\tau$ is scattered.

Proof. We first remark that, if $(\mathcal{P}(X), \delta)$ is a Magari algebra, then the operator $c(A):=A \cup \delta A$ satisfies the Kuratowski axioms of the topological closure: $c \varnothing=\varnothing$, $c(A \cup B)=c A \cup c B, A \subseteq c A, c c A=c A$ (the latter uses the transitivity property of $\delta$ ). This defines a topology $\tau$ on $X$ in which a set $A$ is closed iff $A=c(A)$ iff $\delta A \subseteq A$. If $\delta$ coincides with $d_{\tau}$, this condition is also clearly necessary. So, the required topology is unique. To show that $\delta=d_{\tau}$ indeed, we need an auxiliary lemma.

Lemma 2.5. Suppose $(\mathcal{P}(X), \delta)$ is Magari. Then, for all $x \in X$,

(i) $x \notin \delta(\{x\})$;

(ii) $x \in \delta A \Longleftrightarrow x \in \delta(A \backslash\{x\})$.

Proof. (i) By Axiom M2 we have $\delta\{x\} \subseteq \delta(\{x\} \backslash \delta\{x\})$. If $x \in \delta\{x\}$ then $\delta(\{x\} \backslash \delta\{x\})=\delta \varnothing=\varnothing$. Hence, $\delta\{x\}=\varnothing$, a contradiction.

(ii) $x \in \delta A$ implies $x \in \delta((A \backslash\{x\}) \cup\{x\})=\delta(A \backslash\{x\}) \cup \delta\{x\}$. By (i), $x \notin \delta\{x\}$, hence $x \in \delta(A \backslash\{x\})$. The other implication follows from the monotonicity of $\delta$. $\dashv$

Lemma 2.6. Suppose $(\mathcal{P}(X), \delta)$ is Magari and $\tau$ is the associated topology. Then $\delta=d_{\tau}$.

Proof. Let $d=d_{\tau}$; we show that, for any set $A \subseteq X, d A=\delta A$. Notice that, for any $B, c B=d B \cup B=\delta B \cup B$.

Assume $x \in \delta A$ then

$$
x \in \delta(A \backslash\{x\}) \subseteq c(A \backslash\{x\}) \subseteq d(A \backslash\{x\}) \cup(A \backslash\{x\}) .
$$

Since $x \notin A \backslash\{x\}$, we obtain $x \in d(A \backslash\{x\})$. By the monotonicity of $d, x \in d A$. Similarly, if $x \in d A$ then $x \in d(A \backslash\{x\})$. Hence,

$$
x \in c(A \backslash\{x\})=\delta(A \backslash\{x\}) \cup(A \backslash\{x\}) .
$$

Since $x \notin A \backslash\{x\}$ we obtain $x \in \delta A$. 
From this lemma and Theorem 2.1 we infer that the associated topology $\tau$ must be scattered.

Theorem 2.4 shows that to study a natural set-theoretic interpretation of provability logic means to study the semantics of $\diamond$ as a derivative operation on a scattered topological space.

Derivative semantics of modality was first suggested in the fundamental paper by McKinsey and Tarski [42. See the paper by Kudinov and Shehtman in this volume for a detailed survey of such semantics for general topological spaces. The emphasis in this paper is on the logics related to formal provability. Specifically, scattered spaces (and their generalizations) will play a major role here.

\section{Topological completeness theorems}

Natural examples of scattered topological spaces come from orderings. Two examples will play an important role below.

Let $(X, \prec)$ be a strict partial ordering. The left topology $\tau_{\leftarrow}$ on $(X, \prec)$ is given by all sets $A \subseteq X$ such that $\forall x, y(y \prec x \in A \Rightarrow y \in A)$. We obviously have that $(X, \prec)$ is well-founded iff $\left(X, \tau_{\leftarrow}\right)$ is scattered. The right topology or the upset topology is defined similarly.

This topology is, in general, non-Hausdorff. More natural is the order topology on a linear ordering $(X,<)$, which is generated by all intervals $(\alpha, \beta)$ such that $\alpha, \beta \in X \cup\{ \pm \infty\}$ and $\alpha<\beta$. The order topology refines the left topology and is scattered on any ordinal.

Given a topological space $(X, \tau)$, we shall denote the logic of its derivative algebra $\left(\mathcal{P}(X), d_{\tau}\right)$ by $\log (X, \tau)$, and we use similar notation for the logic of a class of derivative algebras.

The $\operatorname{logic} \log (X, \tau)$ is often equivalently defined in terms of valuations as follows. A valuation on $(X, \tau)$ is any map $v$ from the set of propositional formulas to the powerset of $X$ satisfying the following conditions:

- $v(\varphi \vee \psi)=v(\varphi) \cup v(\psi), v(\neg \varphi)=X \backslash v(\varphi), v(\top)=X, v(\perp)=\varnothing$,

- $v(\diamond \varphi)=d(v(\varphi))$.

One usually writes $X, x \vDash_{v} \varphi$ for $x \in v(\varphi)$. A formula $\varphi$ is valid in $X$ (denoted $X \vDash \varphi)$ if $\forall v, v(\varphi)=X$. Then, $\log (X, \tau)$ coincides with the set of all formulas valid in $(X, \tau)$. Similarly, if $\mathcal{C}$ is a class of spaces, $\log (\mathcal{C})$ is the set of formulas valid in all members $(X, \tau) \in \mathcal{C}$.

Since $\log (\mathcal{C})$ is the logic of a class of Magari algebras, this set of formulas is closed under the rules modus ponens, necessitation and substitution, and contains all theorems of Gödel-Löb logic GL. Thus, $\log (\mathcal{C})$ is a normal modal logic extending $\mathbf{G L}$, for any class $\mathcal{C}$ of scattered spaces.

Topological interpretation of provability logic suggests a question whether GL is complete for any natural class of scattered topological spaces. Leo Esakia [27] has shown that GL coincides with the logic of the class of all scattered topological spaces. In fact, it is sufficient to take finite trees (considered as strict partial orderings) with the associated upset topology.

Theorem 3.1 (Esakia). (i) $\log (\mathcal{C})=\mathbf{G L}$, where $\mathcal{C}$ is the class of all finite irreflexive trees taken with the upset topology. 
(ii) There is a countable scattered space $(X, \tau)$ such that $\log (X, \tau)=\mathbf{G L}$.

We notice that this theorem follows almost immediately from the completeness of GL w.r.t. its Kripke semantics. In fact, if $(X, \prec)$ is a strict partial ordering, then the modal algebra associated with the Kripke frame $(X, \prec)$ is the same as the derivative algebra of $(X, \tau)$ where $\tau$ is its upset topology. This implies that any modal logic of a class of strict partial orders is complete w.r.t. topological semantics.

Esakia's theorem has been strengthened by Abashidze [1] and Blass [18.

Theorem 3.2 (Abashidze, Blass). Consider an ordinal $\Omega \geq \omega^{\omega}$ equipped with the order topology. Then $\log (\Omega)=\mathbf{G L}$.

Thus, GL is complete w.r.t. a natural scattered topological space. In Section 5 we give an alternative proof of this result by utilizing the recursive constructions of finite irreflexive trees and ordinals below $\omega^{\omega}$.

\section{Some useful techniques}

\subsection{Ranks and $d$-maps}

An equivalent characterization of scattered spaces is often given in terms of the following transfinite Cantor-Bendixson sequence of subsets of a topological space $(X, \tau)$ :

- $d_{\tau}^{0} X=X ; \quad d_{\tau}^{\alpha+1} X=d_{\tau}\left(d_{\tau}^{\alpha} X\right)$ and

- $d_{\tau}^{\alpha} X=\bigcap_{\beta<\alpha} d_{\tau}^{\beta} X$ if $\alpha$ is a limit ordinal.

It is easy to show by transfinite induction that, for any $(X, \tau)$, all sets $d_{\tau}^{\alpha} X$ are closed and that $d_{\tau}^{\alpha} X \supseteq d_{\tau}^{\beta} X$ whenever $\alpha \leq \beta$.

Theorem 4.1 (Cantor). $(X, \tau)$ is scattered iff $d_{\tau}^{\alpha} X=\varnothing$, for some ordinal $\alpha$.

Proof. Let $d=d_{\tau}$. If $(X, \tau)$ is scattered then we have $d^{\alpha} X \supset d^{\alpha+1} X$, for each $\alpha$ such that $d^{\alpha} X \neq \varnothing$. By cardinality considerations this yields an $\alpha$ such that $d^{\alpha} X=\varnothing$.

Conversely, suppose $A \subseteq X$ is nonempty. Let $\alpha$ be the least ordinal such that $A \nsubseteq d^{\alpha} X$. Obviously, $\alpha$ cannot be a limit ordinal, hence $\alpha=\beta+1$ for some $\beta$ and there is an $x \in A \backslash d^{\beta+1} X$. Since $A \subseteq d^{\beta} X$, we also have $x \in d^{\beta} X$. Since $x \notin d^{\beta+1} X=d\left(d^{\beta} X\right), x$ is isolated in the relative topology of $d^{\beta} X$, and hence in the relative topology of $A \subseteq d^{\beta} X$.

Call the least $\alpha$ such that $d_{\tau}^{\alpha} X=\varnothing$ the Cantor-Bendixson rank of $X$ and denote it by $\rho_{\tau}(X)$. The rank function $\rho_{\tau}: X \rightarrow$ On is defined by

$$
\rho_{\tau}(x):=\min \left\{\alpha: x \notin d_{\tau}^{\alpha+1}(X)\right\} .
$$

Notice that $\rho_{\tau}$ maps $X$ onto $\rho_{\tau}(X)=\left\{\alpha: \alpha<\rho_{\tau}(X)\right\}$. Also, $\rho_{\tau}(x) \geq \alpha$ iff $x \in d_{\tau}^{\alpha} X$. We omit the subscript $\tau$ whenever there is no danger of confusion.

Example 4.1. Let $\Omega$ be an ordinal equipped with its left topology. Then $\rho(\alpha)=\alpha$, for all $\alpha$. 
Example 4.2. Let $\Omega$ be an ordinal equipped with its order topology. Then $\rho$ is the function $\ell$ defined by

$$
\ell(0)=0 ; \quad \ell(\alpha)=\beta \text { if } \alpha=\gamma+\omega^{\beta}, \text { for some } \gamma, \beta .
$$

By the Cantor normal form theorem, for any $\alpha>0$, such a $\beta$ is uniquely defined.

Let $\left(X, \tau_{X}\right)$ and $\left(Y, \tau_{Y}\right)$ be topological spaces, and let $d_{X}, d_{Y}$ denote the corresponding derivative operators. A map $f: X \rightarrow Y$ is called a $d$-map if $f$ is continuous, open and pointwise discrete, that is, $f^{-1}(y)$ is a discrete subspace of $X$ for each $y \in Y$. $d$-maps are well-known to satisfy the properties expressed in the following lemma (see [16]).

Lemma 4.2. (i) $f^{-1}\left(d_{Y}(A)\right)=d_{X}\left(f^{-1}(A)\right)$, for any $A \subseteq Y$;

(ii) $f^{-1}:\left(\mathcal{P}(Y), d_{Y}\right) \rightarrow\left(\mathcal{P}(X), d_{X}\right)$ is a homomorphism of derivative algebras;

(iii) If $f$ is onto, then $\log \left(X, \tau_{X}\right) \subseteq \log \left(Y, \tau_{Y}\right)$.

In fact, (i) is easy to check directly; (ii) follows from (i) and (iii) follows from (ii). Each of the conditions (i) and (ii) is equivalent to $f$ being a $d$-map.

From lemma 4.2(i) we easily obtain the following corollary by transfinite induction.

Corollary 4.3. Suppose $f: X \rightarrow Y$ is a d-map. Then, for each ordinal $\alpha$, $d_{X}^{\alpha} X=f^{-1}\left(d_{Y}^{\alpha} Y\right)$.

The following lemma states that the rank function, when the ordinals are equipped with their left topology, becomes a $d$-map. It is also uniquely characterized by this property.

Lemma 4.4. Let $\Omega$ be the ordinal $\rho_{\tau}(X)$ taken with its left topology. Then

(i) $\rho_{\tau}: X \rightarrow \Omega$ is an onto $d$-map;

(ii) If $f: X \rightarrow \lambda$ is a d-map, where $\lambda$ is an ordinal with its left topology, then $f(X)=\Omega$ and $f=\rho_{\tau}$.

Proof. Let $\rho$ denote $\rho_{\tau}$.

(i) $\rho$ is continuous, because the set $\rho^{-1}[0, \alpha)=X \backslash d^{\alpha} X$ is open.

$\rho$ being open means that, for each open $U \subseteq X$, whenever $\alpha \in \rho(U)$ and $\beta<\alpha$ one has $\beta \in \rho(U)$. Fix an $x \in U$ such that $\rho(x)=\alpha$. Consider the set $X_{\beta}:=\rho^{-1}(\beta)=d^{\beta} X \backslash d\left(d^{\beta} X\right)$. For any subset $A$ of a scattered space we have $d(A)=d(A \backslash d A)$, hence $d X_{\beta}=d\left(d^{\beta} X\right) \subseteq d^{\alpha} X$. Since $\rho(x)=\alpha$ it follows that $x \in d X_{\beta}$. Hence $U \cap X_{\beta} \neq \varnothing$, that is, $\beta \in \rho(U)$.

$\rho$ being pointwise discrete means $X_{\alpha}=\rho^{-1}(\alpha)$ is discrete, for each $\alpha$. In fact, $X_{\alpha}=d^{\alpha} X \backslash d\left(d^{\alpha} X\right)$ is the set of isolated points of $d^{\alpha} X$. Thus, it cannot help being discrete.

(ii) Since $f$ is a $d$-map, by Corollary 4.3 we obtain that $f^{-1}[\alpha, \lambda)=d^{\alpha} X$, for each $\alpha<\lambda$. Hence, $f^{-1}(\alpha)=\rho^{-1}(\alpha)$, for each $\alpha<\lambda$, that is, $f=\rho$ and $f(X)=\rho(X)=\Omega$.

Let $\rho_{X}$ and $\rho_{Y}$ denote the rank functions of $\left(X, \tau_{X}\right)$ and $\left(Y, \tau_{Y}\right)$, respectively.

Corollary 4.5. If $f: X \rightarrow Y$ is a d-map, then $\rho_{X}=\rho_{Y} \circ f$.

Proof. Clearly, $\rho_{Y} \circ f: X \rightarrow \Omega$ is a $d$-map. Statement (ii) of the previous lemma yields the result. 


\subsection{The d-sum construction}

Given a tree $T$, one can build a new tree by 'plugging in' other trees in place of the maximal points (the 'leaves') of $T$. Similarly, given an ordinal $\alpha$, one can 'plug in' new ordinals $\alpha_{i}$ for each isolated (i.e. non-limit) point $i \in \alpha$ to obtain another ordinal. These constructions of 'summing up' spaces or relational structures 'along' another space or a relational structure are a special case of a general construction, which proved rather useful in its various manifestations for proving topological completeness theorems. Here we present a variant of this construction, called $d$-sum, which can be used to recursively build both finite irreflexive trees and ordinals. We will point out a particular application of $d$-sums by sketching a proof of Abashidze-Blass theorem. For a more substantial application of the $d$-sum construction, in which the summed spaces are homeomorphic to each other, see [5] (where the corresponding construction is called $d$-product).

Definition 4.6. Let $X$ be a topological space and let $\left\{Y_{j} \mid j \in i(X)\right\}$ be a collection of spaces indexed by the set $i(X)$ of isolated points of $X$. We uniquely extend it to the collection $\left\{Y_{j} \mid j \in X\right\}$ by letting $Y_{j}=\{j\}$ for all $j \in d_{\tau} X$.

We define the $d$-sum $\left(Z, \tau_{Z}\right)$ of $\left\{Y_{j}\right\}$ over $X$ (denoted $\left.\sum_{j \in X}^{d} Y_{j}\right)$ as follows. The base set is the disjoint union $Z:=\bigsqcup_{j \in X} Y_{j}$. Define the map $\pi: Z \rightarrow X$ by putting $\pi(y)=j$ whenever $y \in Y_{j}$. Now let the topology $\tau_{Z}$ consist of the sets $V \cup \pi^{-1}(U)$ where $V$ is open in the topological sum $\bigsqcup_{j \in i(X)} Y_{j}$ and $U$ is open in $X$. It is not difficult to check that $\tau_{Z}$ qualifies for a topology.

As an immediate application of this construction we present a way of building finite irreflexive trees (called simply trees for brevity henceforth) recursively using the $d$-sum construction.

Definition 4.7. ( $n$-fork)

Let $\mathfrak{F}_{n}=\left(W_{n}, R_{n}\right)$ be defined as follows: $W_{n}=\left\{r, w_{0}, w_{1}, \ldots, w_{n-1}\right\}$ and $R_{n}=$ $\left\{\left(r, w_{i}\right) \mid 0 \leq i<n\right\}$. We will consider $\mathfrak{F}_{n}$ equipped with the upset topology w.r.t. $R_{n}$.

We view trees, and $n$-forks in particular, as topological spaces.

Definition 4.8. (trees)

(t1) An irreflexive point is a tree.

(t2) An $n$-fork $\mathfrak{F}_{n}$ is a tree.

(t3) The d-sum of trees is a tree.

It is straightforward (if somewhat tedious) to check that trees thus obtained are precisely the finite irreflexive trees. Note that the leaves of a tree are the isolated points in the topology. Therefore, taking the $d$-sum of trees $T_{i}$ over a tree $T$ means simply plugging in $T_{i}$ 's in place of the leaves of $T$. It is worth noting that a tree might be obtained in various different ways as a d-sum of other trees.

In a similar fashion the $d$-sum construction can be applied to ordinals to obtain larger ordinals. For example, it is easy to see that summing up ordinals $\omega^{n}+1$ along the ordinal $\omega+1$ produces the ordinal $\omega^{n+1}+1$. In general, from the wellknown theorem characterizing countable ordinals as countable locally compact scattered Hausdorff spaces, it follows that the $d$-sum of countable ordinals is a countable ordinal. In fact, even more general statement is true. Namely, if one 
takes a family of ordinals $\left(\alpha_{i}\right)_{i \in \beta}$ such that $\alpha_{i}=1$ for limit $i$, then the ordinal $\operatorname{sum} \sum_{i \in \beta} \alpha_{i}$ is an ordinal that is homeomorphic to the d-sum $\sum_{i \in \beta}^{d} \alpha_{i}$. This can be checked directly, by examining the descriptions of neighborhoods in respective topologies. It follows that the $d$-sum of ordinals is an ordinal.

The last paragraph reveals the intrinsic similarity between the inner structure of trees and ordinals. To fix this similarity, we employ the following general lemma showing that $d$-sums, in a way, commute with $d$-maps. More precisely:

Lemma 4.9. Let $X$ and $X^{\prime}$ be two spaces and let $\left\{Y_{j} \mid j \in i(X)\right\}$ and $\left\{Y_{k}^{\prime} \mid k \in\right.$ $\left.i\left(X^{\prime}\right)\right\}$ be collections of spaces indexed by $i(X)$ and $i\left(X^{\prime}\right)$, respectively. Suppose further that $f: X \rightarrow X^{\prime}$ is an onto d-map, and for each $j \in i(X)$ there is an onto $d$-map $f_{j}: Y_{j} \rightarrow Y_{f(j)}^{\prime}$. Then there exists an onto $d$-map $g: \sum_{j \in X}^{d} Y_{j} \rightarrow \sum_{k \in X^{\prime}}^{d} Y_{k}^{\prime}$.

Proof. First note that since $f$ is a $d$-map, $f(j)$ is isolated in $X^{\prime}$ iff $j$ is isolated in $X$. Indeed, by openness of $f$, if $\{j\} \in \tau$, then $\{f(j)\} \in \tau^{\prime}$. Conversely, if $f(j)$ is isolated, then $f^{-1} f(j)$ is both open and discrete, by continuity and pointwise discreteness of $f$. Hence, any point in $f^{-1} f(j)$, and $j$ in particular, is isolated in $X$. For convenience, let us denote $f_{*} \equiv f \uparrow_{d_{\tau} X}$ and $f^{*} \equiv f \uparrow_{i X}$. It follows that $f^{*}: i(X) \rightarrow i\left(X^{\prime}\right)$ and $f_{*}: d_{\tau} X \rightarrow d_{\tau^{\prime}} X^{\prime}$ are well-defined onto maps and $f=f^{*} \cup f_{*}$. Thus, in particular, the space $Y_{f(j)}^{\prime}$ in the formulation of the theorem is well-defined.

Take $g$ to be the set-theoretic union $g=f_{*} \cup \bigcup_{j \in i X} f_{j}$. We show that $g$ is a $d$-map. Let $\pi$ and $\pi^{\prime}$ be the 'projection' maps associated with $\sum_{j \in X}^{d} Y_{j}$ and $\sum_{k \in X^{\prime}}^{d} Y_{k}^{\prime}$, respectively. To show that $g$ is open, take $W=V \cup \pi^{-1}(U) \in \tau_{Z}$. Then $g(W)=g(V) \cup g\left(\pi^{-1}(U)\right)$. That $g(V)$ is open in the topological sum of $Y_{k}^{\prime}$ is clear from the openness of the maps $f_{j}$. Moreover, from the definition of $g$ and the fact that all $f_{j}$ are onto it can be easily deduced that $g\left(\pi^{-1}(U)\right)=\pi^{\prime-1}(f(U))$. Since $f$ is an open map, it follows that $g(W)$ is open in $\tau_{Z}^{\prime}$. To see that $g$ is continuous, take $W^{\prime}=V^{\prime} \cup \pi^{\prime-1}\left(U^{\prime}\right) \in \tau_{Z}^{\prime}$. Then $g^{-1}\left(W^{\prime}\right)=g^{-1}\left(U^{\prime}\right) \cup g^{-1}\left(\pi^{\prime-1}\left(U^{\prime}\right)\right)$. Again, the openness of $g^{-1}\left(U^{\prime}\right)$ is trivial. It is also easily seen that $g^{-1}\left(\pi^{\prime-1}\left(U^{\prime}\right)\right)=$ $\pi^{-1}\left(f^{-1}\left(U^{\prime}\right)\right)$. It follows that $g^{-1}\left(W^{\prime}\right)$ is open in $\tau_{Z}$. To see that $g$ is pointwise discrete is straightforward, given that $f$ and all the $f_{j}$ are pointwise discrete.

In the next section we will use this lemma to prove the Abashidze-Blass theorem.

\section{Proof of Abashidze-Blass theorem}

The topological completeness theorem proved independently by Abashidze and Blass establishes that each non-theorem of GL can be refuted on any ordinal greater than $\omega^{\omega}$. A simplified proof of this result appeared recently in [17, Theorem 3.5]. The crucial part of that proof rests on showing that each tree of depth $n$ is an onto image of an ordinal $\omega^{n}+1$ via a $d$-map [17, Lemma 3.4]. That is probably the simplest and most explicit proof of Abashidze-Blass theorem, however by our approach we aim to illuminate the underlying recursive mechanism, which might offer additional insight. Here is an analog of [17, Lemma 3.4] proved using the $d$-sum construction:

Lemma 5.1. For each tree $T$ there exists a countable ordinal $\alpha<\omega^{\omega}$ and an onto map $f: \alpha \rightarrow T$ such that $f$ is a d-map. 
Proof. [Sketch] The proof proceeds by structural induction. It is clear that the claim is true for a one-point tree. Let us consider now an $n$-fork $\mathfrak{F}_{n}$ and build a $d$-map $f$ from $\omega+1$ onto $\mathfrak{F}_{n}$. Let $f(x)=w_{x \bmod n}$ for $x<\omega$ and let $f(\omega)=r$. It is straightforward to check that $f$ is a $d$-map. That each tree is an image of an appropriate ordinal via a $d$-map now follows from lemma 4.9 using induction on the depth of trees. Indeed, suppose the theorem is true for all trees of depth less than $n$. Take a tree $T$ of depth $n$. It is clear that $T$ can be presented as a $d$-sum of trees of strictly smaller depth in various ways. Using the induction hypothesis, each of the smaller trees is an image of a countable ordinal under a $d$-map. Applying lemma 4.9 and observing that the $d$-sum of countable ordinals is a countable ordinal produces a countable ordinal $\alpha$ and an onto d-map $f: \alpha \rightarrow T$. By corollary 4.5 the rank of $\alpha$ is equal to the rank of $T$, that is, to $n$. It follows that $\alpha<\omega^{\omega}$, which finishes the proof.

One of the ways to present the tree $T$ as a $d$-sum of smaller trees is to 'prune' $T$ immediately after the root. Then $T$ is seen as a $d$-sum of smaller trees along a $k$-fork for some finite $k$. This is precisely the approach usually taken in proving the above lemma. However, the $d$-sum construction allows for greater flexibility. For example, $T$ can be pruned immediately below the leaves, obtaining finitely many forks and a tree of depth $n-1$. Or indeed, $T$ can be pruned anywhere, provided each of the branches of maximal length $n$ is pruned.

And finally, the Abashidze-Blass theorem:

Theorem 5.2 (Abashidze-Blass). The logic of any ordinal $\alpha \geq \omega^{\omega}$ is $\boldsymbol{G L}$.

Proof. Take a non-theorem $\varphi$ of GL. Then $\varphi$ can be refuted on a finite tree $T$. By Lemma 5.1 there exists an ordinal $\beta<\omega^{\omega}$ that maps onto $T$ via a $d$-map. Since $d$-maps reflect satisfiability (the contrapositive of Lemma 4.2 (iii)), $\varphi$ can be refuted on $\beta$. But $\beta$ is an open subspace of $\alpha$. It follows that $\varphi$ can be refuted on $\alpha$.

\section{Topological semantics of linearity axioms}

For a gödelian theory $T$ consider the subalgebra $\mathcal{L}_{T}^{0}$ of $\left(\mathcal{L}_{T}, \diamond_{T}\right)$ generated by $T$. If $\operatorname{ch}\left(\mathcal{L}_{T}, \diamond_{T}\right)=\infty$, then, clearly, $\operatorname{ch}\left(\mathcal{L}_{T}^{0}, \diamond_{T}\right)=\infty$. In fact, the Magari algebra $\left(\mathcal{L}_{T}^{0}, \diamond_{T}\right)$ is known (see [32]) to have the logic GL.3 which is obtained from GL by adding the following axiom:

$$
(.3) \quad \diamond p \wedge \diamond q \rightarrow \diamond(p \wedge q) \vee \diamond(p \wedge \diamond q) \vee \diamond(\diamond p \wedge q)
$$

This is a so called 'linearity axiom' and, as the name suggests, its finite rooted Kripke frames are precisely the finite strict linear orders. Since GL.3 is Kripke complete [23], its topological completeness is almost immediate. However, it is not immediately clear what kind of scattered spaces does the linearity axiom isolate. To characterize GL.3-spaces, let us first simplify the axiom (.3). Let (lin) be the following formula:

$$
\square\left(\square^{+} p \vee \square^{+} q\right) \rightarrow \square p \vee \square q
$$

where $\square^{+} \varphi$ is a shorthand for $\varphi \wedge \square \varphi$.

Lemma 6.1. In $\boldsymbol{G L}$ the schema (.3) is equivalent to (lin). 
Proof. To show that $(\operatorname{lin}) \vdash_{\text {GL }}(.3)$, witness the following syntactic argument. Observe that the dual form of $(\operatorname{lin})$ looks as follows:

$$
\diamond p \wedge \diamond q \rightarrow \diamond\left(\diamond^{+} p \wedge \diamond^{+} q\right)
$$

where $\diamond^{+} \varphi:=\varphi \vee \diamond \varphi$. Furthermore, an instance of the GL axiom looks as follows:

$$
\diamond\left(\diamond^{+} p \wedge \diamond^{+} q\right) \rightarrow \diamond\left(\diamond^{+} p \wedge \diamond^{+} q \wedge \square\left(\square^{+} \neg p \vee \square^{+} \neg q\right)\right) .
$$

By the axiom (lin) we also have:

$$
\square\left(\square^{+} \neg p \vee \square^{+} \neg q\right) \rightarrow(\square \neg p \vee \square \neg q) .
$$

So, using the monotonicity of $\diamond$ we obtain:

$$
\diamond p \wedge \diamond q \rightarrow \diamond\left(\diamond^{+} p \wedge \diamond^{+} q \wedge(\square \neg p \vee \square \neg q)\right)
$$

By Boolean logic we clearly have:

$$
\diamond^{+} p \wedge \diamond^{+} q \leftrightarrow(p \wedge q) \vee(p \wedge \diamond q) \vee(\diamond p \wedge q) \vee(\diamond p \wedge \diamond q) \quad(* *)
$$

and

$$
(\square \neg p \vee \square \neg q) \leftrightarrow \neg(\diamond p \wedge \diamond q) .
$$

Using these, together with the monotonicity of $\diamond$ we finally arrive at:

$$
\diamond p \wedge \diamond q \rightarrow \diamond((p \wedge q) \vee(p \wedge \diamond q) \vee(\diamond p \wedge q)),
$$

which is equivalent to (.3) since $\diamond$ distributes over $\vee$.

To show the converse, we observe that (.3) implies (lin) even in the system $\mathbf{K}$. Indeed, the formula $(*)$, which is the dual form of $($ lin $)$, can be rewritten, using $(* *)$ and the distribution of $\diamond$ over $\vee$ as follows:

$$
\diamond p \wedge \diamond q \rightarrow \diamond(p \wedge q) \vee \diamond(p \wedge \diamond q) \vee \diamond(\diamond p \wedge q) \vee \diamond(\diamond p \wedge \diamond q),
$$

which is clearly a weakening of $(.3)$. Therefore $(.3) \vdash_{\text {GL }}($ lin $)$.

It follows that a scattered space is a GL.3-space iff it validates (lin). To characterize such spaces, consider the following definition.

Definition 6.2. Call a scattered space primal if for each $x \in X$ and $U, V \in \tau$, $\{x\} \cup U \cup V \in \tau$ implies $\{x\} \cup U \in \tau$ or $\{x\} \cup V \in \tau$.

In other words, $X$ is primal if the collection of punctured neighborhoods of each non-isolated point is a prime filter in the Heyting algebra $\tau$.

Theorem 6.3. Let $X$ be a scattered space. Then $X \vDash($ lin $)$ iff $X$ is primal.

Proof. Let $X$ be a scattered space together with a valuation $v$. Let $P:=v(p)$ and $Q:=v(q)$ denote the truth-sets of $p$ and $q$ respectively. Then the truth sets of $\square^{+} p$ and $\square^{+} q$ are $I_{\tau} P$ and $I_{\tau} Q$, where $I_{\tau}$ is the interior operator of $X$. We write $x \vDash \varphi$ for $X, x \vDash_{v} \varphi$ (as introduced in section (3).

Suppose $X$ is primal and for some valuation $x \vDash \square\left(\square^{+} p \vee \square^{+} q\right)$. Then there exists an open neighborhood $W$ of $x$ such that $W \backslash\{x\} \vDash \square^{+} p \vee \square^{+} q$. In other words, $W \backslash\{x\} \subseteq I_{\tau} P \cup I_{\tau} Q$. Let $U=W \cap I_{\tau} P \in \tau$ and $V=W \cap I_{\tau} Q \in \tau$. Then 
$\{x\} \cup U \cup V=W \in \tau$. It follows that either $\{x\} \cup U \in \tau$ or $\{x\} \cup V \in \tau$. Hence $x \vDash \square p$ or $x \vDash \square q$. This proves that $X \vDash($ lin $)$.

Suppose now $X$ is not primal. Then there exist $x \in X$ and $U, V \in \tau$ such that $\{x\} \cup U \cup V \in \tau$, but $\{x\} \cup U \notin \tau$ and $\{x\} \cup V \notin \tau$. Take a valuation such that $P=U$ and $Q=V$. Then clearly $x \vDash \square\left(\square^{+} p \vee \square^{+} q\right)$. However, neither $x \vDash \square p$ nor $x \vDash \square q$ is true. Indeed, if, for example, $x \vDash \square p$, then there exists an open neighborhood $W$ of $x$ such that $W \backslash\{x\} \subseteq P=U$. But then $\{x\} \cup U=W \cup U \in \tau$, which is a contradiction. This shows that $X \not p($ lin $)$.

The primal scattered spaces are very close to maximal scattered spaces of [5]. In fact, each maximal scattered space is primal, but there are primal spaces which are not maximal. It follows that the logic of maximal scattered spaces is GL.3.

\section{GLP-algebras and polymodal provability logic}

A natural generalization of provability logic GL to a language with infinitely many modalities $\langle 0\rangle,\langle 1\rangle, \ldots$ has been introduced as early as in 1986 by Giorgi Japaridze [35]. He interpreted $\langle 1\rangle \varphi$ as an arithmetical statement expressing the $\omega$ consistency of $\varphi$ over a given gödelian theory $T 2$ Similarly, $\langle 2\rangle \varphi$ was interpreted as the consistency of the extension of $T+\varphi$ by two nested applications of $\omega$-rule, and so on.

While the logic of each of the individual modalities $\langle n\rangle$ over Peano arithmetic was known to coincide with GL by a relatively straightforward extension of the Solovay Theorem [19], Japaridze found a complete axiomatization of the joint logic of the modalities $\langle n\rangle$, for all $n \in \omega$. This result involved considerable technical difficulties and lead to one of the first genuine extensions of Solovay's arithmetical fixed-point construction. Later, Japaridze's work has been simplified and extended by Konstantin Ignatiev [34] and George Boolos [20]. In particular, Ignatiev showed that GLP is complete for more general sequences of 'strong' provability predicates in arithmetic and analyzed the variable-free fragment of GLP. Boolos included a treatment of GLP (for the somewhat simpler case of just two modalities) in his popular book on provability logic [21].

More recently, GLP has found interesting applications in proof-theoretic analysis of arithmetic [6, 7, 2, 9] which stimulated some further interest in the study of modal-logical properties of GLP [22, 15, 33, 11. For such applications, the algebraic language appears to be more natural and a different choice of the interpretation of the provability predicates is needed. The relevant structures have been introduced in [6] under the name graded provability algebras.

Recall that an arithmetical formula is called $\Pi_{n}$ if it can be obtained from a formula containing only bounded quantifiers $\forall x \leq t$ and $\exists x \leq t$ by a prefix of $n$ alternating blocks of quantifiers starting from $\forall$. Arithmetical $\Sigma_{n}$-formulas are defined dually.

Let $T$ be a gödelian theory. $T$ is called $n$-consistent if $T$ together with all true arithmetical $\Pi_{n}$-sentences is consistent. (Alternatively, $T$ is $n$-consistent iff every $\Sigma_{n}$-sentence provable in $T$ is true.) Let $n$-Con $(T)$ denote a natural arithmetical

\footnotetext{
${ }^{2} \mathrm{~A}$ gödelian theory $U$ is $\omega$-consistent if its extension by unnested applications of the $\omega$-rule $U^{\prime}:=$ $U+\{\forall x \varphi(x): \forall n U \vdash \varphi(\underline{n})\}$ is consistent.
} 
formula expressing the $n$-consistency of $T$ (it can be defined using the standard $\Pi_{n}$-definition of truth for $\Pi_{n}$-sentences in arithmetic). Since we assume $T$ to be recursively enumerable, it is easy to check that the formula $n$-Con $(T)$ itself belongs to the class $\Pi_{n+1}$.

The $n$-consistency formula induces an operator $\langle n\rangle_{T}$ acting on the Lindenbaum-Tarski algebra $\mathcal{L}_{T}$ :

$$
\langle n\rangle_{T}:[\varphi] \longmapsto[n-\operatorname{Con}(T+\varphi)] .
$$

The dual $n$-provability operators are defined by $[n]_{T} x=\neg\langle n\rangle_{T} x$, for all $x \in \mathcal{L}_{T}$. Since every true $\Pi_{n}$-sentence is assumed to be an axiom for $n$-provability, we notice that every true $\Sigma_{n+1}$-sentence must be $n$-provable. Moreover, this latter fact is formalizable in $T$, so we obtain the following lemma. (By the abuse of notation we denote by $[n]_{T} \varphi$ also the arithmetical formula expressing the $n$-provability of $\varphi$ in T.)

Lemma 7.1. For each true $\Sigma_{n+1}$-formula $\sigma(x)$,

$$
T \vdash \forall x\left(\sigma(x) \rightarrow[n]_{T} \sigma(\underline{x})\right) .
$$

As a corollary we obtain the following basic observation probably due to Smorynski (see [45]).

Proposition 7.2. For each $n \in \omega$, the structure $\left(\mathcal{L}_{T},\langle n\rangle_{T}\right)$ is a Magari algebra.

A proof of this fact consists of verifying the Bernays-Löb derivability conditions for $[n]_{T}$ in $T$ and of deducing from them, in the usual way, an analog of Löb's theorem for $[n]_{T}$.

The structure $\left(\mathcal{L}_{T},\left\{\langle n\rangle_{T}: n \in \omega\right\}\right)$ is called the graded provability algebra of $T$ or the GLP-algebra of T. Apart from the identities inherited from the structure of Magari algebras for each $\langle n\rangle$ it satisfies the following principles, for all $m<n$ :

P1. $\langle m\rangle x \leq[n]\langle m\rangle x$;

P2. $\langle n\rangle x \leq\langle m\rangle x$.

The validity of $\mathrm{P} 1$ follows from Lemma 7.1 , because the formula $\langle m\rangle_{T} \varphi$, for any $\varphi$, belongs to the class $\Pi_{m+1}$. P2 holds, since $\langle n\rangle_{T} \varphi$ asserts the consistency of a stronger theory than $\langle m\rangle_{T} \varphi$, for $m<n$.

In general, we call a GLP-algebra a structure $(M,\{\langle n\rangle: n \in \omega\})$ such that each $(M,\langle n\rangle)$ is a Magari algebra and conditions P1, P2 (that are equivalent to identities) are satisfied for all $x \in M$.

At this point it is worth noticing that $\mathrm{P} 1$ has an equivalent form (modulo the other identities) that has proved to be quite useful for various applications of GLP-algebras.

Lemma 7.3. Modulo the identities of Magari algebras and P2, condition P1 is equivalent to

$\mathrm{P} 1^{\prime} .\langle n\rangle y \wedge\langle m\rangle x=\langle n\rangle(y \wedge\langle m\rangle x)$, for all $m<n$.

Proof. First, we prove $\mathrm{P}^{\prime}$. We have $y \wedge\langle m\rangle x \leq y$, hence

$$
\langle n\rangle(y \wedge\langle m\rangle x) \leq\langle n\rangle y .
$$


Similarly, by P2 and transitivity,

$$
\langle n\rangle(y \wedge\langle m\rangle x) \leq\langle n\rangle\langle m\rangle x \leq\langle m\rangle\langle m\rangle x \leq\langle m\rangle x .
$$

Hence, $\langle n\rangle(y \wedge\langle m\rangle x) \leq\langle n\rangle y \wedge\langle m\rangle x$. In the other direction, by P1,

$$
\langle n\rangle y \wedge\langle m\rangle x \leq\langle n\rangle y \wedge[n]\langle m\rangle x .
$$

However, as in any modal algebra, we also have $\langle n\rangle y \wedge[n] z \leq\langle n\rangle(y \wedge z)$. It follows that

$$
\langle n\rangle y \wedge[n]\langle m\rangle x \leq\langle n\rangle(y \wedge\langle m\rangle x) .
$$

Thus, $\mathrm{P} 1^{\prime}$ is proved.

To infer $\mathrm{P} 1$ from $\mathrm{P} 1^{\prime}$ it is sufficient to prove that

$$
\langle m\rangle x \wedge \neg[n]\langle m\rangle x=\perp .
$$

We have that $\neg[n]\langle m\rangle x=\langle n\rangle \neg\langle m\rangle x$. Therefore, by $\mathrm{P} 1^{\prime}$,

$$
\langle m\rangle x \wedge\langle n\rangle \neg\langle m\rangle x=\langle n\rangle(\neg\langle m\rangle x \wedge\langle m\rangle x)=\langle n\rangle \perp=\perp,
$$

as required.

An equivalent formulation of Japaridze's arithmetical completeness theorem is that any identity of $\left(\mathcal{L}_{T},\left\{\langle n\rangle_{T}: n \in \omega\right\}\right)$ follows from the identities of GLPalgebras [35]. It is somewhat strengthened to the current formulation in [34, 13].

Theorem 7.4 (Japaridze). Suppose $T$ is gödelian, T contains Peano arithmetic, and $\operatorname{ch}\left(\mathcal{L}_{T},\langle n\rangle_{T}\right)=\infty$, for each $n<\omega$. Then, an identity holds in $\left(\mathcal{L}_{T},\left\{\langle n\rangle_{T}\right.\right.$ : $n \in \omega\})$ iff it holds in all GLP-algebras.

We note that the condition $\operatorname{ch}\left(\mathcal{L}_{T},\langle n\rangle_{T}\right)=\infty$, for each $n \in \omega$, is equivalent to $T+n$-Con $(T)$ being consistent, for each $n \in \omega$, and is clearly necessary for the validity of Japaridze's theorem.

The logic of all GLP-algebras can also be axiomatized as a Hilbert-style calculus. The corresponding system GLP was originally introduced by Japaridze. GLP is formulated in the language of propositional logic enriched by modalities $[n]$, for all $n \in \omega$. The axioms of GLP are those of $\mathbf{G L}$, formulated for each $[n]$, as well as the two analogs of $\mathrm{P} 1$ and $\mathrm{P} 2$, for all $m<n$ :

P1. $\langle m\rangle \varphi \rightarrow[n]\langle m\rangle \varphi$;

P2. $[m] \varphi \rightarrow[n] \varphi$.

The inference rules of GLP are modus ponens and $\varphi /[n] \varphi$, for each $n \in \omega$.

For any modal formula $\varphi, \mathbf{G L P} \vdash \varphi$ iff the identity $t_{\varphi}=\top$ holds in all GLPalgebras. Hence, GLP coincides with logic of all GLP-algebras as well as with the logic of the GLP-algebra of $T$, for any theory $T$ such that $T+n$-Con $(T)$ is consistent, for each $n<\omega$. 


\section{GLP-spaces}

Topological semantics for GLP has been first considered in [14. The main difficulty in the modal-logical study of GLP comes from the fact that it is incomplete with respect to its relational semantics; that is, GLP is the logic of no class of frames. Even though a suitable class of relational models for which GLP is sound and complete was developed in [11, these models are sufficiently complicated and not so easy to handle. So, it is natural to consider the generalization of the topological semantics we have for GL. As it turns out, topological semantics provides another natural class of GLP-algebras which is interesting in its own right, as well as because of its analogy with the proof-theoretic GLP-algebras.

As before, we are interested in GLP-algebras of the form $(\mathcal{P}(X),\{\langle n\rangle: n \in$ $\omega\})$ where $\mathcal{P}(X)$ is the boolean algebra of subsets of a given set $X$. Since each $(\mathcal{P}(X),\langle n\rangle)$ is a Magari algebra, the operator $\langle n\rangle$ is the derivative operator with respect to some uniquely defined scattered topology on $X$. Thus, we come to the following definition [14].

A polytopological space $\left(X,\left\{\tau_{n}: n \in \omega\right\}\right)$ is called a GLP-space if the following conditions hold, for each $n \in \omega$ :

D0. $\left(X, \tau_{n}\right)$ is a scattered topological space;

D1. For each $A \subseteq X, d_{\tau_{n}}(A)$ is $\tau_{n+1}$-open;

D2. $\tau_{n} \subseteq \tau_{n+1}$.

We notice that the last two conditions directly correspond to conditions P1 and P2 of GLP-algebras. By a $G L P_{m}$-space we mean a space $\left(X,\left\{\tau_{n}: n<m\right\}\right)$ satisfying conditions D0-D2 for the first $m$ topologies.

Proposition 8.1. (i) If $\left(X,\left\{\tau_{n}: n \in \omega\right\}\right)$ is a GLP-space then the structure $\left(\mathcal{P}(X),\left\{d_{\tau_{n}}: n \in \omega\right\}\right)$ is a GLP-algebra.

(ii) If $(\mathcal{P}(X),\{\langle n\rangle: n \in \omega\})$ is a GLP-algebra, then there are uniquely defined topologies $\left\{\tau_{n}: n \in \omega\right\}$ on $X$ such that $\left(X,\left\{\tau_{n}: n \in \omega\right\}\right)$ is a GLP-space and $\langle n\rangle=d_{\tau_{n}}$, for each $n<\omega$.

Proof. (i) Suppose $\left(X,\left\{\tau_{n}: n \in \omega\right\}\right)$ is a GLP-space. Let $d_{n}:=d_{\tau_{n}}$ denote the corresponding derivative operators and let $\tilde{d}_{n}$ denote its dual $\tilde{d}_{n}(A):=X \backslash d_{n}(X \backslash$ $A)$. By Theorem $2.1\left(\mathcal{P}(X), d_{n}\right)$ is a Magari algebra, for each $n \in \omega$. Notice that $A \in \tau_{n}$ iff $A \subseteq \tilde{d}_{n} A$. If $m<n$ then $d_{m} A \in \tau_{n}$, hence $d_{m} A \subseteq \tilde{d}_{n} d_{m} A$, hence P1 holds. Since $\tau_{n} \subseteq \tau_{n+1}$ we have $d_{n+1} A \subseteq d_{n} A$ hence P2 holds.

(ii) Let $(\mathcal{P}(X),\{\langle n\rangle: n \in \omega\})$ be a GLP-algebra. Since each of the algebras $(\mathcal{P}(X),\langle n\rangle)$ is Magari, by Theorem 2.4 a scattered topology $\tau_{n}$ on $X$ is defined for which $\langle n\rangle=d_{\tau_{n}}$. In fact, we have $U \in \tau_{n}$ iff $U \subseteq[n] U$. We check that conditions D1 and D2 are met.

Suppose $A$ is $\tau_{n}$-closed, that is, $\langle n\rangle A \subseteq A$. Then $\langle n+1\rangle A \subseteq\langle n\rangle A \subseteq A$ by $\mathrm{P} 2$. Hence, $U$ is $\tau_{n+1}$-closed. Thus, $\tau_{n} \subseteq \tau_{n+1}$.

By $\mathrm{P} 1$, for any set $A$ we have $\langle n\rangle A \subseteq[n+1]\langle n\rangle A$. Hence, $d_{\tau_{n}}(A)=\langle n\rangle A \in$ $\tau_{n+1}$. Thus, $\left(X,\left\{\tau_{n}: n \in \omega\right\}\right)$ is a GLP-space. 


\section{Derivative topology and generated GLP- spaces}

To obtain examples of GLP-spaces let us first consider the case of two modalities. The following basic example is due to Esakia (private communication).

Example 9.1. Consider a bitopological space $\left(\Omega ; \tau_{0}, \tau_{1}\right)$ where $\Omega$ is an ordinal, $\tau_{0}$ is its left topology, and $\tau_{1}$ is its order topology. Esakia noticed that this space is a model of the bimodal fragment of GLP, that is, in our terminology, a GLP $_{2^{-}}$ space. In fact, for any $A \subseteq \Omega$ the set $d_{0}(A)=(\min A, \Omega)$ is an open interval, whenever $A$ is not empty. Hence, condition D1 holds (the other two conditions are immediate). Esakia also noticed that such spaces can never be complete for GLP, as the linearity axiom (.3) holds for $\langle 0\rangle$.

In general, to define $\mathrm{GLP}_{n}$-spaces for $n>1$, we introduce an operation $\tau \longmapsto$ $\tau^{+}$on topologies on a given set $X$. This operation plays a central role in the study of GLP-spaces.

Given a topological space $(X, \tau)$, let $\tau^{+}$be the coarsest topology containing $\tau$ such that each set of the form $d_{\tau}(A)$, with $A \subseteq X$, is open in $\tau^{+}$. Thus, $\tau^{+}$ is generated by $\tau$ and $\left\{d_{\tau}(A): A \subseteq X\right\}$. Clearly, $\tau^{+}$is the coarsest topology on $X$ such that $\left(X ; \tau, \tau^{+}\right)$is a $\mathrm{GLP}_{2}$-space. Sometimes we call $\tau^{+}$the derivative topology of $(X, \tau)$.

Getting back to Esakia's example, it is easy to verify that, on any ordinal $\Omega$, the derivative topology of the left topology coincides with the order topology. (In fact, any open interval is an intersection of a downset and an open upset.)

Example 9.2. Even though we are mainly interested in scattered spaces, the derivative topology makes sense for arbitrary spaces. The reader can check that if $\tau$ is the coarsest topology on a set $X$ (whose open sets are just $X$ and $\varnothing$ ), then $\tau^{+}$ is the cofinite topology on $X$ (whose open sets are exactly the cofinite subsets of $X)$. On the other hand, if $\tau$ is the cofinite topology then $\tau^{+}=\tau$. We note that the logic of the cofinite topology on an infinite set is KD45 (see [47]).

For scattered spaces, $\tau^{+}$is always strictly finer than $\tau$, unless $\tau$ is discrete. We present a proof using the language of Magari algebras.

Proposition 9.1. Suppose $(X, \tau)$ is scattered. Then $d_{\tau}(X)$ is not open, unless $d_{\tau}(X)=\varnothing$.

Proof. The set $d_{\tau}(X)$ corresponds to the element $\diamond \top$ in the associated Magari algebra; $d_{\tau}(X)$ being open means $\diamond \top \leq \square \diamond \top$. By Löb's principle we have $\square \diamond \top \leq$ $\square \perp=\neg \diamond \top$. Hence, $\diamond \top \leq \neg \diamond \top$, that is, $\diamond \top=\perp$. This means $d_{\tau}(X)=\varnothing$, as required.

In general, we will see later that $\tau^{+}$can be much finer than $\tau$. Notice that if $\tau$ is $T_{d}$ then each set of the form $d_{\tau}(A)$ is $\tau$-closed. Hence, it will be clopen in $\tau^{+}$. Thus, $\tau^{+}$is obtained by adding to $\tau$ new clopen sets. In particular, $\tau^{+}$will be zero-dimensional if so is $\tau$

Iterating the plus operation delivers us a GLP-space. Let $(X, \tau)$ be a scattered space. Define: $\tau_{0}:=\tau$ and $\tau_{n+1}:=\tau_{n}^{+}$. Then $\left(X,\left\{\tau_{n}: n \in \omega\right\}\right)$ is a GLP-space that will be called the GLP-space generated from $(X, \tau)$, or simply the generated GLP-space.

\footnotetext{
${ }^{3}$ Recall that a topological space is zero-dimensional if it has a base of clopen sets.
} 
Thus, from any scattered space we can always produce a GLP-space in a natural way. The question is whether this space will be nontrivial, that is, whether we can guarantee that the topologies $\tau_{n}$ are non-discrete.

In fact, the next observation shows that for many natural $\tau$ already the topology $\tau^{+}$will be discrete. Recall that a topological space $X$ is first-countable if every point $x \in X$ has a countable basis of open neighborhoods.

Proposition 9.2. If $(X, \tau)$ is Hausdorff and first-countable, then $\tau^{+}$is discrete.

Proof. It is easy to see that if $(X, \tau)$ is first-countable and Hausdorff, then every point $a \in d_{\tau}(X)$ is a (unique) limit point of a countable sequence of points $A=\left\{a_{n}\right\}_{n \in \omega}$. Hence, there is a set $A \subseteq X$ such that $d_{\tau}(A)=\{a\}$. By D1 this means that $\{a\}$ is $\tau^{+}$-open.

Thus, if $\tau$ is the order topology on a countable ordinal, then $\tau^{+}$is discrete. The same holds, for example, if $\tau$ is the (non-scattered) topology of the real line.

We remark that the left topology $\tau$ on any countable ordinal $>\omega$ yields an example of a first-countable space such that $\tau^{+}$is non-discrete. In the following section we will also see that, if $\tau$ is the order topology on any ordinal $>\omega_{1}$, then $\tau^{+}$is non-discrete $\left(\omega_{1}\right.$ is its least non-isolated point). However, we do not have any topological characterization of spaces $(X, \tau)$ such that $\tau^{+}$is discrete. (See, however, Proposition 10.3, which provides a characterization in terms of $d$-reflection.)

It is a natural question to ask what kind of separation properties is $\tau^{+}$guaranteed to have, for an arbitrary (scattered) topology $\tau$. In fact, for $\tau^{+}$we can infer a bit more separation than for an arbitrary scattered topology. Recall that a topological space $X$ is $T_{1}$ if, for any two different points $a, b \in X$, there is a open set $U$ such that $a \in U$ and $b \notin U$.

Proposition 9.3. Let $(X, \tau)$ be any topological space. Then $\left(X, \tau^{+}\right)$is $T_{1}$.

Proof. Let $a, b \in X, a \neq b$. We must show that there is an open set $A$ such that $a \in A$ and $b \notin A$. Consider the set $B:=d_{\tau}(\{b\})$, which is open in $\tau^{+}$. If $a \in B$ then we are done, because $b \notin d_{\tau}(\{b\})=B$. Otherwise, if $a \notin B$, neither does $a$ belong to the closure of $\{b\}$ (which is simply $\{b\} \cup d_{\tau}(\{b\})$ ). It follows that the complement of $\{b\} \cup B$ is the required open set.

The following example shows that, in general, $\tau^{+}$need not always be Hausdorff.

Example 9.3. Let $(X, \prec)$ be a strict partial ordering on $X:=\omega \cup\{a, b\}$ where $\omega$ is taken with its natural order, $a$ and $b$ are $\prec$-incomparable, and $n \prec a, b$, for all $n \in \omega$. Let $\tau$ be the left topology on $(X, \prec)$. Since $\prec$ is well-founded, $\tau$ is scattered.

Notice that $d_{\tau}(A)=\{x \in X: \exists y \in A y \prec x\}$. Hence, if $A$ intersects $\omega$, then $d_{\tau}(A)$ contains an end-segment of $\omega$. Otherwise, $d_{\tau}(A)=\varnothing$. It follows that a base of open neighborhoods of $a$ in $\tau^{+}$consists of sets of the form $I \cup\{a\}$ where $I$ is an end-segment of $\omega$. Similarly, sets of the form $I \cup\{b\}$ are a base of open neighborhoods of $b$. But any two such sets have a non-empty intersection. 


\section{$10 d$-reflection}

In the next section we are going to describe in some detail the GLP-space generated from the left topology on the ordinals. Strikingly, we will see that it naturally leads to some of the central notions of combinatorial set theory, such as Mahlo operation and stationary reflection. In fact, part of our analysis can be easily stated using the language of modal logic for arbitrary generated GLP-spaces. In this section we provide a necessary setup and characterize the topologies of a generated GLPspace in terms of what we call $d$-reflection. 4

Throughout this section we will assume that $(X, \tau)$ is a $T_{d}$ space and let $d=d_{\tau}$. Thus, $(\mathcal{P}(X), d)$ is a K4-algebra.

Definition 10.1. A point $a \in X$ is called $d$-reflexive if $a \in d X$ and, for each $A \subseteq X$,

$$
a \in d A \Rightarrow a \in d(d A) .
$$

In modal logic terms this means that the following formula is valid at $a \in X$, for any evaluation of the variable $p$ :

$$
X, a \vDash \diamond \top \wedge(\diamond p \rightarrow \diamond \diamond p) .
$$

Similarly, a point $a \in X$ is called $m$-fold $d$-reflexive if $a \in d X$ and, for each $A_{1}, \ldots, A_{m} \subseteq X$

$$
a \in d A_{1} \cap \cdots \cap d A_{m} \Rightarrow a \in d\left(d A_{1} \cap \cdots \cap d A_{m}\right) .
$$

2 -fold $d$-reflexive points will also be called doubly $d$-reflexive points. Modal logically, $a \in X$ is doubly $d$-reflexive iff

$$
X, a \vDash \diamond \top \wedge(\diamond p \wedge \diamond q \rightarrow \diamond(\diamond p \wedge \diamond q))
$$

Lemma 10.2. Each doubly d-reflexive point $x \in X$ is $m$-fold $d$-reflexive, for any finite $m$.

Proof. The argument goes by induction on $m \geq 2$. Suppose $x \in d A_{1} \cap \cdots \cap$ $d A_{m+1}$, then $x \in d A_{1} \cap \cdots \cap d A_{m}$ and $x \in d A_{m+1}$. By induction hypothesis,

$$
x \in d\left(d A_{1} \cap \cdots \cap d A_{m}\right)
$$

and by 2-fold reflection

$$
x \in d\left(d\left(d A_{1} \cap \cdots \cap d A_{m}\right) \cap d A_{m+1}\right) .
$$

However, by $T_{d}$ property

$$
d\left(d A_{1} \cap \cdots \cap d A_{m}\right) \subseteq d A_{1} \cap \cdots \cap d A_{m},
$$

hence

$$
x \in d\left(d A_{1} \cap \cdots \cap d A_{m} \cap d A_{m+1}\right),
$$

as required.

\footnotetext{
${ }^{4}$ Curiously, the reader may notice that the notion of reflection principle as used in provability logic and formal arithmetic matches very nicely the notions such as stationary reflection in set theory. (As far as we know, the two terms have evolved completely independently from one another.)
} 
Proposition 10.3. Let $(X, \tau)$ be a $T_{d}$-space. A point $x \in X$ is doubly d-reflexive iff $x$ is a limit point of $\left(X, \tau^{+}\right)$.

Proof. For the (if) direction, we give an argument in the algebraic format. In fact, it is sufficient to show the following inequality in the algebra of $(X, \tau)$, for any elements $p, q \subseteq X$ :

$$
\langle 1\rangle \top \wedge\langle 0\rangle p \wedge\langle 0\rangle q \leq\langle 0\rangle(\langle 0\rangle p \wedge\langle 0\rangle q) .
$$

Notice that by Proposition 7.3 ,

$$
\langle 1\rangle \top \wedge\langle 0\rangle p=\langle 1\rangle(\top \wedge\langle 0\rangle p)=\langle 1\rangle\langle 0\rangle p .
$$

Hence, we obtain using $\mathrm{P} 1^{\prime}$ once again:

$$
\langle 1\rangle \top \wedge\langle 0\rangle p \wedge\langle 0\rangle q=\langle 1\rangle\langle 0\rangle p \wedge\langle 0\rangle q=\langle 1\rangle(\langle 0\rangle p \wedge\langle 0\rangle q) .
$$

The latter formula can be weakened to $\langle 0\rangle(\langle 0\rangle p \wedge\langle 0\rangle q)$ by P2, as required.

For the (only if) direction, it is sufficient to show that each doubly $d$-reflexive point of $(X, \tau)$ is a limit point of $\tau^{+}$. Suppose $x$ is doubly $d$-reflexive. By Lemma 10.2, $x$ is $m$-fold $d$-reflexive.

Any basic open subset of $\tau^{+}$has the form

$$
U:=A_{0} \cap d A_{1} \cap \cdots \cap d A_{m},
$$

where $A_{0} \in \tau$. Assume $x \in U$, we have to find a point $y \neq x$ such that $y \in U$.

Since $x \in d A_{1} \cap \cdots \cap d A_{m}$, by $m$-fold $d$-reflexivity we obtain $x \in d\left(d A_{1} \cap \cdots \cap\right.$ $\left.d A_{m}\right)$. Since $A_{0}$ is an open neighborhood of $x$, there is a $y \in A_{0}$ such that $y \neq x$ and $y \in d A_{1} \cap \cdots \cap d A_{m}$. Hence $y \in U$ and $y \neq x$, as required.

Let $d^{+}$denote the derivative operator associated with $\tau^{+}$. We obtain the following characterization of derived topology in terms of neighborhoods.

Proposition 10.4. A subset $U \subseteq X$ contains a $\tau^{+}{ }_{\text {-neighborhood of } x \in X \text { iff one }}$ of the following two cases holds:

(i) $x$ is not doubly d-reflexive and $x \in U$;

(ii) $x$ is doubly d-reflexive and there is an $A \in \tau$ and a $B$ such that $x \in A \cap d B \subseteq$ $U$.

Proof. Since (i) ensures that $x$ is $\tau^{+}$-isolated, each condition is clearly sufficient for $U$ to contain a $\tau^{+}$-neighborhood of $x$. To prove the converse, assume that $U$ contains a $\tau^{+}$-neighborhood of $x$. This means

$$
x \in A \cap d A_{1} \cap \cdots \cap d A_{m} \subseteq U,
$$

for some $A, A_{1}, \ldots, A_{m}$ with $A \in \tau$.

If $x$ is $\tau^{+}$-isolated, condition (i) holds. Otherwise, $x \in d^{+} X$. Let $B:=$ $d A_{1} \cap \cdots \cap d A_{m}$. Since $B$ is closed in $\tau$ we have $d B \subseteq B$, hence $A \cap d B \subseteq U$. It remains us to show that $x \in A \cap d B$. By Lemma 7.3, $B \cap d^{+} X=d^{+} B \subseteq d B$. Hence, $x \in A \cap B \cap d^{+} X \subseteq A \cap d B$.

Remark 10.1. Since in (ii) of the previous lemma $A$ is open, we have $A \cap d B=$ $A \cap d(A \cap B)$, for any $B$. Hence, we can assume $B \subseteq A$. 
Corollary 10.5. Let $(X, \tau)$ be a $T_{d}$-space. Then, for all $x \in X$ and $A \subseteq X$, $x \in d^{+} A$ iff the following two conditions hold:

(i) $x$ is doubly d-reflexive;

(ii) For all $B \subseteq X, x \in d B \Rightarrow x \in d(A \cap d B)$.

The second condition is similar to $x$ being $d$-reflexive, however $d B$ is now required to reflect from $x$ to a point of $A$.

Proof. The fact that (i) and (ii) are necessary is proved using Proposition 10.3 and the inequality

$$
d^{+} A \cap d B=d^{+}(A \cap d B) \subseteq d(A \cap d B) .
$$

We prove that (i) and (ii) are sufficient. Assume $x \in U \in \tau^{+}$. By Proposition 10.4 we can assume that $U$ has the form $V \cap d B$ where $V \in \tau$. By (ii), from $x \in d B$ we obtain $x \in d(A \cap d B)$. Hence, there is a $y \neq x$ such that $y \in V$ and $y \in A \cap d B$. It follows that $y \in A$ and $y \in V \cap d B=U$.

\section{The ordinal GLP-space}

Here we discuss the GLP-space generated from the left topology on the ordinals, that is, the GLP-space $\left(\Omega ;\left\{\tau_{n}: n \in \omega\right\}\right)$ with $\tau_{0}$ the left topology on $\Omega$ and $\tau_{n+1}=\tau_{n}^{+}$, for each $n \in \omega$. It is convenient to think of $\Omega$ as the class of all ordinals, even though some readers might feel safer with $\Omega$ being a large fixed ordinal. Our basic findings are summarized in the following table, to which we provide extended comments below.

The rows of the table correspond to topologies $\tau_{n}$. The first column contains the name of the topology (the first two are standard, the third one is introduced in [14, the fourth one is introduced here). The second column indicates the first limit point of $\tau_{n}$, which is denoted $\theta_{n}$. The last column describes the derivative operator associated with $\tau_{n}$. We note that $\theta_{3}$ is a large cardinal which is sometimes referred to as the first cardinal reflecting for pairs of stationary sets (see below), but we know no special notation for this cardinal.

\begin{tabular}{l|c|c|l} 
& name & $\theta_{n}$ & $d_{n}(A)$ \\
\hline$\tau_{0}$ & left & 1 & $\{\alpha: A \cap \alpha \neq \varnothing\}$ \\
\hline$\tau_{1}$ & order & $\omega$ & $\{\alpha \in \operatorname{Lim}: A \cap \alpha$ is unbounded in $\alpha\}$ \\
\hline$\tau_{2}$ & club & $\omega_{1}$ & $\{\alpha: \operatorname{cf}(\alpha)>\omega$ and $A \cap \alpha$ is stationary in $\alpha\}$ \\
\hline$\tau_{3}$ & Mahlo & $\theta_{3}$ & $\ldots \ldots$
\end{tabular}

We have already seen that the derivative topology of the left topology is exactly the order topology. Therefore, basic facts related to the first two rows of the table are rather clear. We turn to the next topology $\tau_{2}$.

\subsection{Club topology}

To characterize $\tau_{2}$ we apply Proposition 10.4 hence it is useful to see what corresponds to the notion of doubly $d$-reflexive point of the interval topology.

Recall that the cofinality $\operatorname{cf}(\alpha)$ of a limit ordinal $\alpha$ is the least order type of a cofinal subset of $\alpha ; \operatorname{cf}(\alpha):=0$ if $\alpha \notin \operatorname{Lim}$. An ordinal $\alpha$ is regular if $\operatorname{cf}(\alpha)=\alpha$. 
Lemma 11.1. For any ordinal $\alpha, \alpha$ is $d_{1}$-reflexive iff $\alpha$ is doubly $d_{1}$-reflexive iff $\operatorname{cf}(\alpha)>\omega$.

Proof. $d_{1}$-reflexivity of $\alpha$ implies $\operatorname{cf}(\alpha)>\omega$. In fact, $d_{1}$-reflexivity of $\alpha$ means that $\alpha \in \operatorname{Lim}$ and, for all $A \subseteq \alpha$, if $A$ is cofinal in $\alpha$, then $d_{1}(A)$ is cofinal in $\alpha$. If $\operatorname{cf}(\alpha)=\omega$ then there is an increasing sequence $\left(\alpha_{n}\right)_{n \in \omega}$ such that $\sup \left\{\alpha_{n}: n \in\right.$ $\omega\}=\alpha$. Then, for $A:=\left\{\alpha_{n}: n \in \omega\right\}$ we obviously have $d_{1}(A)=\{\alpha\}$, hence $A$ violates the reflexivity property.

Now we show that $\operatorname{cf}(\alpha)>\omega$ implies $\alpha$ is doubly $d_{1}$-reflexive. Suppose $\operatorname{cf}(\alpha)>$ $\omega$ and $A, B \subseteq \alpha$ are both cofinal in $\alpha$. We show that $d_{1} A \cap d_{1} B$ is cofinal in $\alpha$. Assume $\beta<\alpha$. Using the cofinality of $A, B$ we can construct an increasing sequence $\left(\gamma_{n}\right)_{n \in \omega}$ above $\beta$ such that $\gamma_{n} \in A$, for even $n$, and $\gamma_{n} \in B$ for odd $n$. Let $\gamma:=\sup \left\{\gamma_{n}: n<\omega\right\}$. Obviously, both $A$ and $B$ are cofinal in $\gamma$ whence $\gamma \in d_{1} A \cap d_{1} B$. Since $\operatorname{cf}(\alpha)>\omega$ and $\operatorname{cf}(\gamma)=\omega$, we have $\gamma<\alpha$.

Corollary 11.2. Limit points of $\tau_{2}$ are exactly the ordinals of uncountable cofinality.

It turns out that topology $\tau_{2}$ is strongly related to the well-known concept of club filter in set theory.

Let $\alpha$ be a limit ordinal. A subset $C \subseteq \alpha$ is called a $c l u b$ in $\alpha$ if $C$ is closed in the order topology of $\alpha$ and unbounded in $\alpha$. The filter on $\alpha$ generated by all clubs in $\alpha$ is called the club filter.

Proposition 11.3. Assume $\operatorname{cf}(\alpha)>\omega$. The following statements are equivalent:

(i) $U$ contains a $\tau_{2}$-neighborhood of $\alpha$;

(ii) There is a $B \subseteq \alpha$ such that $\alpha \in d_{1} B \subseteq U$;

(iii) $\alpha \in U$ and $U$ contains a club in $\alpha$;

(iv) $\alpha \in U$ and $U \cap \alpha$ belongs to the club filter on $\alpha$.

Proof. Statement (ii) implies (iii), since $\alpha \cap d_{1} B$ is a club in $\alpha$, whenever $\alpha \in d_{1} B$. Statement (iii) implies (iv), for obvious reasons.

Statement (iv) implies (i). If $C$ is a club in $\alpha$, then $C \cup\{\alpha\}$ contains a $\tau_{2^{-}}$ neighborhood $d_{1} C$ of $\alpha$. Indeed, $d_{1} C$ is $\tau_{2}$-open, contains $\alpha$, and $d_{1} C \subseteq C \cup\{\alpha\}$ since $C$ is $\tau_{1}$-closed in $\alpha$.

Statement (i) implies (ii). Assume $U$ contains a $\tau_{2}$-neighborhood of $\alpha$. Since $\operatorname{cf}(\alpha)>\omega$, by Lemma 11.1 and Proposition 10.4 there is an $A \in \tau_{1}$ and a $B_{1}$ such that $\alpha \in A \cap d_{1} B_{1} \subseteq U$. Since $A$ is a $\tau_{1}$-neighborhood of $\alpha$, by Proposition 10.4 there are $A_{0} \in \tau_{0}$ and a $B_{0}$ such that $\alpha \in A_{0} \cap d_{0} B_{0}$. Since $\tau_{0}$ is the left topology, we can assume that $A_{0}$ is the minimal $\tau_{0}$-neighborhood $[0, \alpha]$ of $\alpha$. Besides, we have $\alpha \in d_{0} B_{0} \cap d_{1} B_{1}=d_{1}\left(B_{1} \cap d_{0} B_{0}\right) \subseteq U$. Since $[0, \alpha]$ is $\tau_{1}$-clopen, $d_{1}(C \cap \alpha)=[0, \alpha] \cap d_{1} C$ for any $C$, so we can take $B_{1} \cap d_{0} B_{0} \cap \alpha$ for $B$.

Definition 11.4. The club topology on $\Omega$ is the unique topology such that

- If $\operatorname{cf}(\alpha) \leq \omega$ then $\alpha$ is an isolated point;

- If $\operatorname{cf}(\alpha)>\omega$, then, for any $U \subseteq \Omega, U$ contains a neighborhood of $\alpha$ iff $\alpha \in U$ and $U$ contains a club in $\alpha$.

As an immediate corollary of Proposition 11.3 we obtain 
Theorem 11.5. $\tau_{2}$ coincides with the club topology.

We remark that the above theorem saves us the little work of verifying that the club topology is, indeed, a topology.

The derivative operation for the club topology is also well-known in set theory. Recall the following definition for $\operatorname{cf}(\alpha)>\omega$.

A subset $A \subseteq \alpha$ is called stationary in $\alpha$ if $A$ intersects every club in $\alpha$. Observe that this happens exactly when $\alpha$ is a limit point of $A$ in $\tau_{2}$, so

$$
d_{2}(A)=\{\alpha: \operatorname{cf}(\alpha)>\omega \text { and } A \cap \alpha \text { is stationary in } \alpha\} .
$$

The map $d_{2}$ is usually called the Mahlo operation (see [36] where $d_{2}$ is denoted $\mathrm{Tr}$ ). Its main significance is associated with the notion of Mahlo cardinal, one of the basic examples of large cardinals in set theory. Let Reg denote the class of regular cardinals; the ordinals in $d_{2}(\mathrm{Reg})$ are called weakly Mahlo cardinals. Their existence implies the consistency of ZFC, as well as the consistency of ZFC together with the assertion 'inaccessible cardinals exist.'

Now we turn to topology $\tau_{3}$.

\subsection{Stationary reflection and Mahlo topology}

Since the open sets of $\tau_{3}$ are generated by the Mahlo operation, we call $\tau_{3}$ Mahlo topology. It turns out to be intrinsically connected with the concept of stationary reflection, an extensively studied concept in set theory.

We adopt the following terminology. An ordinal $\lambda$ is called reflecting if $\operatorname{cf}(\lambda)>$ $\omega$ and, whenever $A$ is stationary in $\lambda$, there is an $\alpha<\lambda$ such that $A \cap \alpha$ is stationary in $\alpha$. Similarly, $\lambda$ is doubly reflecting if $\operatorname{cf}(\lambda)>\omega$ and whenever $A, B$ are stationary in $\lambda$ there is an $\alpha<\lambda$ such that both $A \cap \alpha$ and $B \cap \alpha$ are stationary in $\alpha$.

Mekler and Shelah's notion of reflection cardinal [43] is somewhat more general than the one given here, however it has the same consistency strength. Reflection for pairs of stationary sets has been introduced by Magidor [41. Since $d_{2}$ coincides with the Mahlo operation, we immediately obtain the following statement.

Proposition 11.6. (i) $\lambda$ is reflecting iff $\lambda$ is $d_{2}$-reflexive;

(ii) $\lambda$ is doubly reflecting iff $\lambda$ is doubly $d_{2}$-reflexive;

(iii) $\lambda$ is a non-isolated point in $\tau_{3}$ iff $\lambda$ is doubly reflecting.

Together with the next proposition this yields a characterization of Mahlo topology in terms of neighborhoods.

Proposition 11.7. Suppose $\lambda$ is doubly reflecting. For any subset $U \subseteq \Omega$, the following conditions are equivalent:

(i) $U$ contains a $\tau_{3}$-neighborhood of $\lambda$;

(ii) $\lambda \in U$ and there is $a B \subseteq \lambda$ such that $\lambda \in d_{2} B \subseteq U$;

(iii) $\lambda \in U$ and there is a $\tau_{2}$-closed (in the relative topology of $\lambda$ ) stationary $C \subseteq \lambda$ such that $C \subseteq U$.

Notice that the notion of $\tau_{2}$-closed stationary $C$ in (iii) is the analog of the notion of club for the $\tau_{2}$-topology. 
Proof. Condition (ii) implies (iii). Since $\lambda$ is reflecting, if $\lambda \in d_{2} B$ then $\lambda \in$ $d_{2} d_{2} B$, that is, $\lambda \cap d_{2} B$ is stationary in $\lambda$. So we can take $C:=\lambda \cap d_{2} B$.

Condition (iii) implies (ii). If $C$ is $\tau_{2}$-closed and stationary in $\lambda$, then $d_{2} C \subseteq$ $C \cup\{\lambda\} \subseteq U$ and $\lambda \in d_{2} C$. Thus, $\lambda \cap d_{2} C$ can be taken for $B$.

Condition (ii) implies (i). If (ii) holds, $U$ contains a subset of the form $d_{2} B$. The latter is $\tau_{3}$-open and contains $\lambda$, thus, is a neighborhood of $\lambda$.

For the converse direction, we note that by Proposition $10.4 U$ contains a subset of the form $A \cap d_{2} B$ where $A \in \tau_{2}, B \subseteq A$ and $\lambda \in A \cap d_{2} B$. Since $A$ is a $\tau_{2}$-neighborhood of $\lambda$, by Theorem 11.5 there is a set $B_{1}$ such that $\lambda \in$ $[0, \lambda] \cap d_{1} B_{1} \subseteq A$. Then,

$$
\lambda \in[0, \lambda] \cap d_{1} B_{1} \cap d_{2} B=[0, \lambda] \cap d_{2}\left(B \cap d_{1} B_{1}\right) .
$$

Since $[0, \lambda]$ is clopen, we obtain $\lambda \in d_{2} C$ with $C:=B \cap d_{1} B_{1} \cap \lambda$.

Reflecting and doubly reflecting cardinals are large cardinals in the sense that their existence implies consistency of ZFC. They have been studied by Mekler and Shelah [43] and Magidor [41] who investigated their consistency strength and related them to some other well-known large cardinals. By a result of Magidor, the existence of a doubly reflecting cardinal is equiconsistent with the existence of a weakly compact cardinal.5 More precisely, the following proposition holds.

Proposition 11.8. (i) If $\lambda$ is weakly compact then $\lambda$ is doubly reflecting.

(ii) (Magidor) If $\lambda$ is doubly reflecting then $\lambda$ is weakly compact in $L$.

Here, the first item is well-known and easy. Magidor originally proved the analog of the second item for $\lambda=\aleph_{2}$ and stationary sets of ordinals of countable cofinality in $\aleph_{2}$. However, it has been remarked by Mekler and Shelah [43] that essentially the same proof yields the stated claim 6

Corollary 11.9. Assertion " $\tau_{3}$ is non-discrete" is equiconsistent with the existence of a weakly compact cardinal.

Corollary 11.10. If ZFC is consistent then it is consistent with ZFC that $\tau_{3}$ is discrete and hence that $\mathbf{G L P}_{3}$ is incomplete w.r.t. any ordinal space.

Recall that $\theta_{n}$ denotes the first non-isolated point of $\tau_{n}$ (in the space of all ordinals). We have: $\theta_{0}=1, \theta_{1}=\omega, \theta_{2}=\omega_{1}, \theta_{3}$ is the first doubly reflecting cardinal.

ZFC does not know much about the location of $\theta_{3}$, however the following facts are interesting.

- $\theta_{3}$ is regular, but not a successor of a regular cardinal;

- While weakly compact cardinals are non-isolated, $\theta_{3}$ need not be weakly compact: If infinitely many supercompact cardinals exist, then there is a model where $\aleph_{\omega+1}$ is doubly reflecting (Magidor [41]);

- If $\theta_{3}$ is a successor of a singular cardinal, then some very strong large cardinal hypothesis must be consistent (Woodin cardinals).

\footnotetext{
${ }^{5}$ Weakly compact cardinals are the same as $\Pi_{1}^{1}$-indescribable cardinals, see below.

${ }^{6}$ The first author thanks J. Cummings for clarifying this.
} 


\subsection{Further topologies}

Further topologies of the ordinal GLP-space do not seem to have prominently occured in set-theoretic work. They yield some large cardinal notions, for the statement that $\tau_{n}$ is non-discrete (or, equivalently, the statement that $\theta_{n}$ exists) implies the existence of a doubly reflecting cardinal, for any $n>2$. We do not know whether cardinals $\theta_{n}$ coincide with any of the standard large cardinal notions.

Here we give a sufficient condition for the topology $\tau_{n+2}$ to be non-discrete. We show that, if there exists a $\Pi_{n}^{1}$-indescribable cardinal, then $\tau_{n+2}$ is non-discrete.

Let $Q$ be a class of second order formulas over the standard first order settheoretic language enriched by a unary predicate $R$. We assume $Q$ to contain at least the class of all first order formulas (denoted $\Pi_{0}^{1}$ ). We shall consider standard models of that language of the form $\left(V_{\alpha}, \in, R\right)$, where $\alpha$ is an ordinal, $V_{\alpha}$ is the $\alpha$-th class in the cumulative hierarchy, and $R$ is a subset of $V_{\alpha}$.

We would like to give a definition of $Q$-indescribable cardinals in topological terms. They can then be defined as follows.

Definition 11.11. For any sentence $\varphi \in Q$ and any $R \subseteq V_{\kappa}$, let $U_{\kappa}(\varphi, R)$ denote the set

$$
\left\{\alpha \leq \kappa:\left(V_{\alpha}, \in, R \cap V_{\alpha}\right) \vDash \varphi\right\} .
$$

The $Q$-describable topology $\tau_{Q}$ on $\Omega$ is generated by a subbase consisting of sets $U_{\kappa}(\varphi, R)$, for all $\kappa \in \Omega, \varphi \in Q$, and all $R \subseteq V_{\kappa}$.

As an exercise, the reader can check that the intervals $(\alpha, \beta]$ are open in any $\tau_{Q}$ (consider $R=\{\alpha\}$ and $\varphi=\exists x(x \in R)$ ). The main strength of the $Q$-describable topology, however, comes from the fact that a second order variable $R$ is allowed to occur in $\varphi$. So, a lot of subsets of $\Omega$ that can be 'described' in this way are open in $\tau_{Q}$.

Let $d_{Q}$ denote the derivative operator for $\tau_{Q}$. An ordinal $\kappa$ is called $Q$ indescribable if it is a limit point of $\tau_{Q}$. In other words, $\kappa$ is $Q$-indescribable iff $\kappa \in d_{Q}($ On $)$ iff $\kappa \in d_{Q}(\kappa)$.

It is not difficult to show that, whenever $Q$ is any of the classes $\Pi_{n}^{1}$, the sets $U_{\kappa}(\varphi, R)$ actually form a base for $\tau_{Q}$. Hence, our definition of $\Pi_{n}^{1}$-indescribable cardinals is equivalent to the standard one given in [37]: $\kappa$ is $Q$-indescribable iff, for all $R \subseteq V_{\kappa}$ and all sentences $\varphi \in Q$,

$$
\left(V_{\kappa}, \in, R\right) \vDash \varphi \Rightarrow \exists \alpha<\kappa\left(V_{\alpha}, \in, R \cap V_{\alpha}\right) \vDash \varphi .
$$

A weakly compact cardinal can be defined as the $\Pi_{1}^{1}$-indescribable one. The well-known fact that weakly compact cardinals are doubly reflecting can be somewhat more generally stated as follows: the Mahlo topology $\tau_{3}$ is contained in $\tau_{\Pi_{1}^{1}}$. We omit a short proof, because we are going to prove a more general proposition suggested to the first author by Philipp Schlicht (see [10]).

Proposition 11.12. For any $n \geq 0, \tau_{n+2}$ is contained in $\tau_{\Pi_{n}^{1}}$.

Proof. We shall show that, for each $n$, there is a $\Pi_{n}^{1}$-formula $\varphi_{n+1}(R)$ such that

$$
\kappa \in d_{n+1}(A) \Longleftrightarrow\left(V_{\kappa}, \in, A \cap \kappa\right) \vDash \varphi_{n+1}(R) .
$$

This implies that, for each $\kappa \in d_{n+1}(A)$, the set $U_{\kappa}\left(\varphi_{n+1}, A \cap \kappa\right)$ is a $\tau_{\Pi_{n}^{1}}$-open subset of $d_{n+1}(A)$ containing $\kappa$. Hence, each $d_{n+1}(A)$ is $\tau_{\Pi_{n}^{1}}$-open. Since $\tau_{n+2}$ is 
generated over $\tau_{n+1}$ by the open sets of the form $d_{n+1}(A)$ for various $A$, we have $\tau_{n+2} \subseteq \tau_{\Pi_{n}^{1}}$.

We prove $(* *)$ by induction on $n$. For $n=0$, notice that $\kappa \in d_{1}(A)$ iff $(\kappa \in \operatorname{Lim}$ and $A \cap \kappa$ is unbounded in $\kappa$ ) iff

$$
\left(V_{\kappa}, \in, A \cap \kappa\right) \vDash \forall \alpha \exists \beta(R(\beta) \wedge \alpha<\beta) .
$$

For the induction step recall that, by Corollary 10.5, $\kappa \in d_{n+1}(A)$ iff

(i) $\kappa$ is doubly $d_{n}$-reflexive;

(ii) $\forall Y \subseteq \kappa\left(\kappa \in d_{n}(Y) \rightarrow \exists \alpha<\kappa\left(\alpha \in A \wedge \alpha \in d_{n}(Y)\right)\right.$.

By the induction hypothesis, for some $\varphi_{n}(R) \in \Pi_{n-1}^{1}$, we have

$$
\alpha \in d_{n}(A) \Longleftrightarrow\left(V_{\alpha}, \in, A \cap \alpha\right) \vDash \varphi_{n}(R) .
$$

Hence, part (ii) is equivalent to

$$
\left(V_{\kappa}, \in, A \cap \kappa\right) \vDash \forall Y \subseteq \mathrm{On}\left(\varphi_{n}(Y) \rightarrow \exists \alpha\left(R(\alpha) \wedge \varphi_{n}^{V_{\alpha}}(Y \cap \alpha)\right)\right) .
$$

Here, $\varphi^{V_{\alpha}}$ means the relativization of all quantifiers in $\varphi$ to $V_{\alpha}$. We notice that $V_{\alpha}$ is first order definable, hence the complexity of $\varphi_{n}^{V_{\alpha}}$ remains in the class $\Pi_{n-1}^{1}$. So, the resulting formula is $\Pi_{n}^{1}$.

To treat part (i) we recall that $\kappa$ is doubly $d_{n}$-reflexive iff $\kappa \in d_{n}(\mathrm{On})$ and

$$
\forall Y_{1}, Y_{2} \subseteq \kappa\left(\kappa \in d_{n}\left(Y_{1}\right) \cap d_{n}\left(Y_{2}\right) \rightarrow \exists \alpha<\kappa \alpha \in d_{n}\left(Y_{1}\right) \cap d_{n}\left(Y_{2}\right)\right) .
$$

Similarly to the above, using the induction hypothesis this can be rewritten as a $\Pi_{n}^{1}$-formula.

Corollary 11.13. If there is a $\Pi_{n}^{1}$-indescribable cardinal, then $\tau_{n+2}$ has a nonisolated point.

Corollary 11.14. If there is a cardinal which is $\Pi_{n}^{1}$-indescribable, for each $n$, then all $\tau_{n}$ are non-discrete.

We know that $\theta_{3}$ need not be weakly compact in some models of ZFC. Hence, the condition of the existence of $\Pi_{n}^{1}$-indescribable cardinals is not a necessary one for the nontriviality of the topologies $\tau_{n+2}$, in general. However, the two statements are equiconsistent, as has been recently shown by Bagaria, Magidor, and Sakai in [4.

\section{Topological completeness results}

As in the case of the unimodal language (cf Section [3), one can ask two basic questions:

- Is GLP complete w.r.t. the class of all GLP-spaces?

- Is GLP complete w.r.t. some fixed natural GLP-spaces?

In the unimodal case, both questions received positive answers due to Esakia and Abashidze-Blass, respectively. Now the situation is more complicated. 


\subsection{Topological completeness of GLP}

The first question was initially studied by Beklemishev, Bezhanishvili and Icard in [14. In this paper only some partial results were obtained. It was proved that the bimodal system $\mathbf{G L P}_{2}$ is complete w.r.t. $\mathrm{GLP}_{2}$-spaces of the form $\left(X, \tau, \tau^{+}\right)$, where $X$ is a well-founded partial ordering and $\tau$ is its left topology. A proof of this result was based on the Kripke model techniques coming from [11.

Already at that time it was clear that these techniques cannot be immediately generalized to $\mathrm{GLP}_{3}$-spaces, since the third topology $\tau^{++}$on such orderings is sufficiently similar to the club topology. From the results of Andreas Blass described below it was known that some stronger set-theoretic assumptions would be needed to prove the completeness w.r.t. such topologies. Moreover, without any large cardinal assumptions it was not even known whether a GLP-space with a non-discrete third topology could exist at all.

First examples of GLP-spaces in which all topologies are non-discrete are constructed in [5]. In the same paper also the much stronger fact of topological completeness of GLP w.r.t. the class of all (countable, Hausdorff) GLP-spaces is established.

Theorem 12.1. (i) $\log (\mathcal{C})=\mathbf{G L P}$, where $\mathcal{C}$ is the class of all GLP-spaces.

(ii) There is a countable Hausdorff GLP-space $X$ such that $\log (X)=$ GLP.

In fact, $X$ is the ordinal $\varepsilon_{0}$ equipped with a sequence of topologies refining the order topology. However, these topologies cannot be first-countable and are, in fact, defined using non-constructive methods such as Zorn's lemma. In this sense, it is not an example of a natural GLP-space.

A proof of this theorem introduces the techniques of maximal rank-preserving and limit-maximal extensions of scattered spaces. It falls outside the present survey (see [5]).

\subsection{Completeness w.r.t. the ordinal GLP-space}

The question whether GLP is complete w.r.t. some natural GLP-space is still open. Some partial results concerning the GLP-space generated from the order topology on the ordinals are described below. Here, we call this space the ordinal GLP-space. (The space described in the previous section is clearly not an exact model of GLP, as the left topology validates the linearity axiom.)

As we know from Corollary 11.10, it is consistent with ZFC that the Mahlo topology is discrete. Hence, it is consistent that GLP is incomplete w.r.t. the ordinal GLP-space. However, is it consistent that GLP be complete? To this question we do not know a full answer. A pioneering work has been done by Andreas Blass [18] who studied the question of completeness of the Gödel-Löb logic GL w.r.t. a semantics equivalent to the topological interpretation w.r.t. the club topology $\tau_{2}$. He used the language of filters rather than that of topological spaces, as is more common in set theory.

Theorem 12.2 (Blass). (i) If $V=L$ and $\Omega \geq \aleph_{\omega}$, then $\mathbf{G L}$ is complete w.r.t. $\left(\Omega, \tau_{2}\right)$.

(ii) If there is a weakly Mahlo cardinal, there is a model of ZFC in which GL is incomplete w.r.t. $\left(\Omega, \tau_{2}\right)$, for any $\Omega$. 
A corollary of (i) is that the statement "GL is complete w.r.t. $\tau_{2}$ " is consistent with ZFC (provided ZFC is consistent). In fact, instead of $V=L$ Blass used the so-called square principle for all $\aleph_{n}, n<\omega$, which holds in $L$ by the results of Ronald Jensen. A proof of (i) is based on an interesting combinatorial construction using the techniques of splitting stationary sets.

A proof of (ii) is much easier. It uses a model of Harrington and Shelah in which $\aleph_{2}$ is reflecting for stationary sets of ordinals of countable cofinality [31. Assuming Mahlo cardinals exist, they have shown that the following statement holds in some model of ZFC:

If $S$ is a stationary subset of $\aleph_{2}$ such that $\forall \alpha \in S \operatorname{cf}(\alpha)=\omega$, then there is a $\beta<\alpha$ (of cofinality $\omega_{1}$ ) such that $S \cap \beta$ is stationary in $\beta$.

In fact, this statement can be expressed in the language of modal logic. First, we remark that this principle implies its generalization to all ordinals $\lambda$ of cofinality $\aleph_{2}$ (consider an increasing continuous function mapping $\aleph_{2}$ to a club in $\lambda$ ). Second, we remark that, for the club topology, the formula $\diamond^{n} \top$ represents the class ordinals of cofinality at least $\aleph_{n}$. This is a straightforward generalization of Lemma 11.1. Thus, the formula $\square^{3} \perp \wedge \diamond^{2} \top$ represents the subclass of $\Omega$ consisting of ordinals of cofinality $\omega_{2}$.

Hence, the above reflection principle amounts to the validity of the following modal formula:

$$
\square^{3} \perp \wedge \diamond^{2} \top \wedge \diamond(p \wedge \square \perp) \rightarrow \diamond^{2}(p \wedge \square \perp) .
$$

In fact, if the left hand side is valid in $\lambda$, then $\operatorname{cf}(\lambda)=\omega_{2}$ and the interpretation of $p \wedge \square \perp$ is a set $S$ consisting of ordinals of countable cofinality such that $S \cap \lambda$ is stationary in $\lambda$. The right hand side just states that this set reflects. Thus, formula $(*)$ is valid in $\left(\Omega, \tau_{2}\right)$, for any $\Omega$. Since this formula is clearly not provable in $\mathbf{G L}$, the topological completeness fails for $\left(\Omega, \tau_{2}\right)$.

Thus, Blass managed to give an exact consistency strength of the statement "GL is incomplete w.r.t. $\tau_{2} "$.

Corollary 12.3. "GL is incomplete w.r.t. $\tau_{2}$ " is consistent iff it is consistent that Mahlo cardinals exist.

It is possible to generalize these results to the case of bimodal logic $\mathbf{G L P}_{2}$ [12]. The situation remains essentially unchanged, although a proof of Statement (i) of Blass's theorem needs considerable adaptation.

Theorem 12.4. If $V=L$ and $\Omega \geq \aleph_{\omega}$, then $\mathbf{G L P}_{2}$ is complete w.r.t. $\left(\Omega ; \tau_{1}, \tau_{2}\right)$.

\section{Topologies for the variable-free fragment of GLP}

A natural topological model for the variable-free fragment of GLP has been introduced by Thomas Icard [33. It is not a GLP-space, thus, not a model of the full GLP. However, it is sound and complete for the variable-free fragment of GLP. It gives a convenient tool for the study of this fragment, which plays an important role in proof-theoretic applications of the polymodal provability logic. Here, we give a simplified presentation of Icard's polytopological space. 
Let $\Omega$ be an ordinal and let $\ell: \Omega \rightarrow \Omega$ denote the rank function for the order topology on $\Omega$. It is easy to check that $\ell(0)=0$ and $\ell(\alpha)=\beta$ whenever $\alpha=\gamma+\omega^{\beta}$. By the Cantor normal form theorem, such a $\beta$ is uniquely defined for each ordinal $\alpha>0$. We define $\ell^{0}(\alpha)=\alpha$ and $\ell^{k+1}(\alpha)=\ell \ell^{k}(\alpha)$.

Icard's topologies $v_{n}$, for each $n \in \omega$, are defined as follows. Let $v_{0}$ be the left topology, and let $v_{n}$ be generated by $v_{0}$ and all sets of the form

$$
U_{\beta}^{m}:=\left\{\alpha \in \Omega: \ell^{m}(\alpha)>\beta\right\}
$$

for $m<n$.

Clearly, $v_{n}$ is an increasing sequence of topologies. In fact, $v_{1}$ is the standard interval topology. We let $d_{n}$ and $\rho_{n}$ denote the derivative operator and the rank function for $v_{n}$, respectively. We have the following characterizations.

Lemma 13.1. (i) $\ell:\left(\Omega, v_{n+1}\right) \rightarrow\left(\Omega, v_{n}\right)$ is a d-map;

(ii) $v_{n+1}$ is the coarsest topology $\nu$ on $\Omega$ such that $\nu$ contains the interval topology and $\ell:(\Omega, \nu) \rightarrow\left(\Omega, v_{n}\right)$ is continuous;

(iii) $\ell^{n}$ is the rank function of $v_{n}$, that is, $\rho_{n}=\ell^{n}$;

(iv) $v_{n+1}$ is generated by $v_{n}$ and $\left\{d_{n}^{\alpha+1}(\Omega): \alpha<\rho_{n}(\Omega)\right\}$.

Proof. (i) The map $\ell:\left(\Omega, v_{n+1}\right) \rightarrow\left(\Omega, v_{n}\right)$ is continuous. In fact, $\ell^{-1}[0, \beta)$ is open in the order topology $v_{1}$, since $\ell:\left(\Omega, v_{1}\right) \rightarrow\left(\Omega, v_{0}\right)$ is its rank function, hence a $d$-map. Also, if $m<n$ then $\ell^{-1}\left(U_{\beta}^{m}\right)=U_{\beta}^{m+1}$, hence it is open in $v_{n+1}$.

The map $\ell$ is open. Notice that $v_{n+1}$ is generated by $v_{1}$ and some sets of the form $\ell^{-1}(U)$ where $U \in v_{n}$. A base of $v_{n+1}$ consists of sets of the form $V \cap \ell^{-1}(U)$, for some $V \in v_{1}$ and $U \in v_{n}$. We have $\ell\left(V \cap \ell^{-1}(U)\right)=\ell(V) \cap U . \ell(V)$ is $v_{0}$-open, since $\ell:\left(\Omega, v_{1}\right) \rightarrow\left(\Omega, v_{0}\right)$ is a $d$-map and $V \in v_{1}$. Hence, the image of any basic open in $v_{n+1}$ is open in $v_{n}$.

The map $\ell$ is pointwise discrete, since $\ell^{-1}\{\alpha\}$ is discrete in the order topology $v_{1}$, hence in $v_{n+1}$.

(ii) By (i), $\ell:\left(\Omega, v_{n+1}\right) \rightarrow\left(\Omega, v_{n}\right)$ is continuous, hence $\nu \subseteq v_{n+1}$. On the other hand, if $\ell:(\Omega, \nu) \rightarrow\left(\Omega, v_{n}\right)$ is continuous, then $\ell^{-1}\left(U_{\beta}^{m}\right) \in \nu$, for each $m<n$. Therefore $U_{\beta}^{m} \in \nu$, for all $m$ such that $1 \leq m \leq n$. Since $\nu$ also contains the interval topology, we have $v_{n+1} \subseteq \nu$.

(iii) By (i), we have that $\rho_{n} \circ \ell$ is a $d$-map from $\left(\Omega, v_{n+1}\right)$ to $\left(\Omega, v_{0}\right)$. Hence, it coincides with the rank function for $v_{n+1}, \rho_{n+1}=\rho_{n} \circ \ell$. The claim follows by an easy induction on $n$.

(iv) Notice that, by (iii),

$$
d_{n}^{\beta+1}(\Omega)=\left\{\alpha \in \Omega: \rho_{n}(\alpha)>\beta\right\}=\left\{\alpha \in \Omega: \ell^{n}(\alpha)>\beta\right\}=U_{\beta}^{n} .
$$

Obviously, $v_{n+1}$ is generated by $v_{n}$ and $U_{\beta}^{n}$, for all $\beta$. Hence, the claim.

We call Icard space a polytopological space of the form $\left(\Omega ; v_{0}, v_{1}, \ldots\right)$. Icard originally considered just $\Omega=\varepsilon_{0}$. We are going to give an alternative proof of the following theorem [33].

Theorem 13.2 (Icard). Let $\varphi$ be a variable-free GLP-formula.

(i) If $\mathbf{G L P} \vdash \varphi$, then $\left(\Omega ; v_{0}, v_{1}, \ldots\right) \vDash \varphi$.

(ii) If $\Omega \geq \varepsilon_{0}$ and $\mathbf{G L P} \nvdash \varphi$, then $\left(\Omega ; v_{0}, v_{1}, \ldots\right) \not \models \varphi$. 
Proof. Within this proof we abbreviate $\left(\Omega ; v_{0}, v_{1}, \ldots\right)$ by $\Omega$. To prove part (i) we first remark that all topologies $v_{n}$ are scattered, hence all axioms of GLP except for P1 are valid in $\Omega$. Moreover, $\log (\Omega)$ is closed under the inference rules of GLP. Thus, we only have to show that the variable-free instances of axiom P1 are valid in $\Omega$. This is sufficient, because any derivation of a variable-free formula in GLP can be replaced by a derivation in which only the variable-free formulas occur (replace all the variables by the constant $T$ ).

Let $\varphi$ be a variable-free formula. We denote by $\varphi^{*}$ its uniquely defined interpretation in $\Omega$. The validity of an instance of $\mathrm{P} 1$ for $\varphi$ amounts to the fact that $d_{m}\left(\varphi^{*}\right)$ is open in $v_{n}$ whenever $m<n$. Thus, we have to prove the following proposition.

Proposition 13.3. For any variable-free formula $\varphi, d_{n}\left(\varphi^{*}\right)$ is open in $v_{n+1}$.

Let $\varphi^{+}$denote the result of replacing in $\varphi$ each modality $\langle n\rangle$ by $\langle n+1\rangle$. We need the following auxiliary claim.

Lemma 13.4. If $\varphi$ is variable-free, then $\ell^{-1}\left(\varphi^{*}\right)=\left(\varphi^{+}\right)^{*}$.

Proof. This goes by induction on the build-up of $\varphi$. The cases of constants and boolean connectives are easy. Suppose $\varphi=\langle n\rangle \psi$. We notice that since $\ell:\left(\Omega, v_{n+1}\right) \rightarrow\left(\Omega, v_{n}\right)$ is a $d$-map, we have $\ell^{-1}\left(d_{n}(A)\right)=d_{n+1}\left(\ell^{-1}(A)\right)$, for any $A \subseteq \Omega$. Therefore,

$$
\ell^{-1}\left(\varphi^{*}\right)=\ell^{-1}\left(d_{n}\left(\psi^{*}\right)\right)=d_{n+1}\left(\ell^{-1}\left(\psi^{*}\right)\right)=d_{n+1}\left(\left(\psi^{+}\right)^{*}\right)=\left(\varphi^{+}\right)^{*},
$$

as required.

We prove Proposition 13.3 in two steps. First, we show that it holds for a subclass of variable-free formulas called ordered formulas. Then we show that any variable-free formula is equivalent in $\Omega$ to an ordered one.

A formula $\varphi$ is called ordered, if no modality $\langle m\rangle$ occurs within the scope of $\langle n\rangle$ in $\varphi$ for any $m<n$. The height of $\varphi$ is the index of its maximal modality.

Lemma 13.5. If $\langle n\rangle \varphi$ is ordered, then $d_{n}\left(\varphi^{*}\right)$ is open in $v_{n+1}$.

Proof. This goes by induction on the height of $\langle n\rangle \varphi$. If it is 0 , then $n=0$. If $n=0$, the claim is obvious, since $d_{0}(A)$ is open in $v_{1}$, for any $A \subseteq \Omega$. If $n>0$, since $\langle n\rangle \varphi$ is ordered, we observe that $\langle n\rangle \varphi$ has the form $(\langle n-1\rangle \psi)^{+}$, for some $\psi$. The height of $\langle n-1\rangle \psi$ is less than that of $\langle n\rangle \varphi$. Hence, by the induction hypothesis, $(\langle n-1\rangle \psi)^{*} \in v_{n}$. Since $\ell:\left(\Omega, v_{n+1}\right) \rightarrow\left(\Omega, v_{n}\right)$ is continuous, we conclude that $\ell^{-1}(\langle n-1\rangle \psi)^{*}$ is open in $v_{n+1}$. By Lemma 13.4, this set coincides with $(\langle n\rangle \varphi)^{*}=d_{n}\left(\varphi^{*}\right)$.

Lemma 13.6. Any variable-free formula $\varphi$ is equivalent in $\Omega$ to an ordered one.

Proof. We argue by induction on the complexity of $\varphi$. The cases of boolean connectives and constants are easy. Suppose $\varphi$ has the form $\langle n\rangle \psi$, where we can assume $\psi$ to be in disjunctive normal form $\psi=\bigvee_{i} \bigwedge_{j} \pm\left\langle n_{i j}\right\rangle \psi_{i j}$. By the induction hypothesis, we may assume all the subformulas $\left\langle n_{i j}\right\rangle \psi_{i j}$ (and $\psi$ itself) ordered. Since $\langle n\rangle$ commutes with disjunction, it will be sufficient to show that, for each $i$, the formula

$$
\langle n\rangle \bigwedge_{j} \pm\left\langle n_{i j}\right\rangle \psi_{i j}
$$


can be ordered.

By Lemma 13.5 each set $\left(\left\langle n_{i j}\right\rangle \psi_{i j}\right)^{*}$ is open in $v_{n}$, whenever $n_{i j}<n$. Being a derived set, it is also closed in $v_{n_{i j}}$ and hence in $v_{n}$. Thus, all such sets are clopen.

If $U$ is open then $d(A \cap U)=d(A) \cap U$, for any topological space. In particular, for any $A \subseteq \Omega$ and $n_{i j}<n$,

$$
d_{n}\left(A \cap\left( \pm\left\langle n_{i j}\right\rangle \psi_{i j}\right)^{*}\right)=d_{n}(A) \cap\left( \pm\left\langle n_{i j}\right\rangle \psi_{i j}\right)^{*} .
$$

This allows us to bring all the conjuncts $\pm\left\langle n_{i j}\right\rangle \psi_{i j}$ from under the $\langle n\rangle$ modality in $(*)$. The resulting conjunction is ordered.

This concludes the proof of Proposition 13.3 and thereby of Part (i).

To prove Part (ii), we shall rely on the following fundamental lemma about the variable-free fragment of GLP. For a purely syntactic proof of this lemma we refer the reader to [6, 8].

A variable-free formula $A$ is called a word if it is built-up from $\top$ only using connectives of the form $\langle n\rangle$, for any $n \in \omega$. We write $A \vdash B$ for GLP $\vdash A \rightarrow B$.

Lemma 13.7. (i) Every variable-free formula is equivalent in GLP to a boolean combination of words;

(ii) For any words $A$ and $B$, either $A \vdash\langle 0\rangle B$, or $B \vdash\langle 0\rangle A$, or $A$ and $B$ are equivalent;

(iii) Conjunction of words is equivalent to a word.

We prove Part (ii) of Theorem 13.2 in a series of lemmas. First, we show that any word is satisfiable in $\Omega$ provided $\Omega \geq \varepsilon_{0}$.

Lemma 13.8. For any word $A, \varepsilon_{0} \in A^{*}$.

Proof. We know that $\rho_{n}\left(\varepsilon_{0}\right)=\ell^{n}\left(\varepsilon_{0}\right)=\varepsilon_{0}$. Hence, $\varepsilon_{0} \in d_{n}(\Omega)$, for each $n$. Assume $n$ exceeds all the indices of modalities in $A$ and $A=\langle m\rangle B$. By Proposition 13.3 the set $B^{*}$ is open in $v_{n}$. By the induction hypothesis $\varepsilon_{0} \in B^{*}$. Hence, $\varepsilon_{0} \in d_{n}\left(B^{*}\right) \subseteq d_{m}\left(B^{*}\right)=A^{*}$. This proves the claim.

Applying this lemma to the word $\langle 0\rangle A$ we obtain the following corollary.

Corollary 13.9. For every word $A$, there is an $\alpha<\varepsilon_{0}$ such that $\alpha \in A^{*}$.

Let $\min \left(A^{*}\right)$ denote the least ordinal $\alpha \in \Omega$ such that $\alpha \in A^{*}$.

Lemma 13.10. For any words $A, B$, if $A \nvdash B$ then $\min \left(A^{*}\right) \notin B^{*}$.

Proof. If $A \nvdash B$ then, by Lemma 13.7 (ii), $B \vdash\langle 0\rangle A$. Therefore, by the soundness of GLP in $\Omega, B^{*} \subseteq d_{0}\left(A^{*}\right)$. It follows that for each $\beta \in B^{*}$ there is an $\alpha \in A^{*}$ such that $\alpha<\beta$. Thus, $\min \left(A^{*}\right) \notin B^{*}$.

Now we are ready to prove Part (ii). Assume $\varphi$ is variable-free and GLP $\nvdash \varphi$. By Lemma 13.7 (i) we can assume that $\varphi$ is a boolean combination of words. Writing $\varphi$ in conjunctive normal form we observe that it is sufficient to prove the claim only for formulas $\varphi$ of the form $\bigwedge_{i} A_{i} \rightarrow \bigvee_{j} B_{j}$, where $A_{i}$ and $B_{j}$ are words. Moreover, $\bigwedge_{i} A_{i}$ is equivalent to a single word $A$.

Since GLP $\nvdash \varphi$ we have $A \nvdash B_{j}$, for each $j$. Let $\alpha=\min \left(A^{*}\right)$. By Lemma 13.10 we have $\alpha \notin B_{j}^{*}$, for each $j$. Hence, $\alpha \notin\left(\bigvee_{j} B_{j}\right)^{*}$ and $\alpha \notin \varphi^{*}$. This means that $\Omega \not \models \varphi^{*}$. 


\section{Further results}

Topological semantics of polymodal provability logic has been extended to the language with transfinitely many modalities. A logic $\mathbf{G L P} \mathbf{P}_{\Lambda}$ having modalities $[\alpha]$, for all ordinals $\alpha<\Lambda$, is introduced in [8]. It was intended for the proof-theoretic analysis of predicative theories and is currently being actively investigated for that purpose.

David Fernandez and Joost Joosten undertook a thorough study of the variable-free fragment of that logic mostly in connection with the arising ordinal notation systems (see [24, 26] for a sample). In particular, they found a suitable generalization of Icard's polytopological space and showed that it is complete for that fragment [25]. Fernandez [29] also proved topological completeness of the full $\mathbf{G L P}_{\Lambda}$ by generalizing the results in [5].

The ordinal GLP-space is easily generalized to transfinitely many topologies $\left(\tau_{\alpha}\right)_{\alpha<\Lambda}$ by letting $\tau_{0}$ be the left topology, $\tau_{\alpha+1}:=\tau_{\alpha}^{+}$and, for limit ordinals $\lambda, \tau_{\lambda}$ be the topology generated by all $\tau_{\alpha}$ such that $\alpha<\lambda$. This space is a natural model of $\mathbf{G L P}_{\Lambda}$ and has been studied quite recently by Joan Bagaria 3 and further by Bagaria, Magidor and Sakai [4. In particular, the three authors proved that in $L$ the limit points of $\tau_{n+2}$ are $\Pi_{n}^{1}$-indescribable cardinals. The question posed in [14] whether the non-discreteness of $\tau_{n+2}$ is equiconsistent with the existence of $\Pi_{n}^{1}$-indescribable cardinals still seems to be open.

\section{Acknowledgement}

The first author was supported by the Russian Foundation for Basic Research (RFBR), Russian Presidential Council for Support of Leading Scientific Schools, and the Swiss-Russian cooperation project STCP-CH-RU "Computational proof theory".

The second author was supported by the Shota Rustaveli National Science Foundation grant \#FR/489/5-105/11 and the French-Georgian grant CNRSSRNSF \#4135/05-01.

\section{References}

[1] M. Abashidze. Ordinal completeness of the Gödel-Löb modal system. In Intensional logics and the logical structure of theories, pages 49-73. Metsniereba, Tbilisi, 1985. In Russian.

[2] S.N. Artemov and L.D. Beklemishev. Provability logic. In D. Gabbay and F. Guenthner, editors, Handbook of Philosophical Logic, 2nd ed., volume 13, pages 229-403. Kluwer, Dordrecht, 2004.

[3] J. Bagaria. Topologies on ordinals and the completeness of polymodal provability logic. In preparation, 2012.

[4] J. Bagaria, M. Magidor, and H. Sakai. Reflection and indescribability in the constructible universe. Manuscript, 2012.

[5] L. Beklemishev and D. Gabelaia. Topological completeness of the provability logic GLP. Preprint arXiv:1106.5693v1 [math.LO], 2011. 
[6] L.D. Beklemishev. Provability algebras and proof-theoretic ordinals, I. Annals of Pure and Applied Logic, 128:103-123, 2004.

[7] L.D. Beklemishev. Reflection principles and provability algebras in formal arithmetic. Uspekhi Matematicheskikh Nauk, 60(2):3-78, 2005. In Russian. English translation in: Russian Mathematical Surveys, 60(2): 197-268, 2005.

[8] L.D. Beklemishev. Veblen hierarchy in the context of provability algebras. In P. Hájek, L. Valdés-Villanueva, and D. Westerståhl, editors, Logic, Methodology and Philosophy of Science, Proceedings of the Twelfth International Congress, pages 65-78. Kings College Publications, London, 2005. Preprint: Logic Group Preprint Series 232, Utrecht University, June 2004.

[9] L.D. Beklemishev. The Worm Principle. In Z. Chatzidakis, P. Koepke, and W. Pohlers, editors, Lecture Notes in Logic 27. Logic Colloquium '02, pages 75-95. AK Peters, 2006. Preprint: Logic Group Preprint Series 219, Utrecht University, March 2003.

[10] L.D. Beklemishev. On GLP-spaces. Manuscript, http://www.mi.ras.ru/ bekl/Papers/glp-sp.pdf, 2009.

[11] L.D. Beklemishev. Kripke semantics for provability logic GLP. Annals of Pure and Applied Logic, 161:756-774, 2010. Preprint: Logic Group Preprint Series 260, University of Utrecht, November 2007. http://preprints.phil.uu.nl/lgps/.

[12] L.D. Beklemishev. Ordinal completeness of bimodal provability logic GLB. In N. Bezhanishvili et al., editor, Logic, Language and Computation, TbiLLC 2009. Lecture Notes in Artificial Intelligence, number 6618, pages 1-15, 2011.

[13] L.D. Beklemishev. A simplified proof of the arithmetical completeness theorem for the provability logic GLP. Trudy Matematicheskogo Instituta imeni V.A.Steklova, 274(3):32-40, 2011. In Russian. English translation: Proceedings of the Steklov Institute of Mathematics, 274(3):25-33, 2011.

[14] L.D. Beklemishev, G. Bezhanishvili, and T. Icard. On topological models of GLP. In R. Schindler, editor, Ways of Proof Theory, Ontos Mathematical Logic, pages 133-152. Ontos Verlag, Heusendamm bei Frankfurt, Germany, 2010. Preprint: Logic Group Preprint Series 278, University of Utrecht, August 2009, http://preprints.phil.uu.nl/lgps/.

[15] L.D. Beklemishev, J. Joosten, and M. Vervoort. A finitary treatment of the closed fragment of Japaridze's provability logic. Journal of Logic and Computation, 15(4):447-463, 2005.

[16] G. Bezhanishvili, L. Esakia, and D. Gabelaia. Some results on modal axiomatization and definability for topological spaces. Studia Logica, 81:325-355, 2005 .

[17] G. Bezhanishvili and P. Morandi. Scattered and hereditarily irresolvable spaces in modal logic. Archive, 49(3):343-365, 2010.

[18] A. Blass. Infinitary combinatorics and modal logic. Journal of Symbolic Logic, 55(2):761-778, 1990.

[19] G. Boolos. The Unprovability of Consistency: An Essay in Modal Logic. Cambridge University Press, Cambridge, 1979.

[20] G. Boolos. The analytical completeness of Dzhaparidze's polymodal logics. Annals of Pure and Applied Logic, 61:95-111, 1993. 
[21] G. Boolos. The Logic of Provability. Cambridge University Press, Cambridge, 1993.

[22] L. Carlucci. Worms, gaps and hydras. Mathematical Logic Quarterly, 51(4):342-350, 2005.

[23] A. Chagrov and M. Zakharyaschev. Modal Logic. Clarendon Press, Oxford, 1997.

[24] D. Fernández-Duque and J. J. Joosten. Kripke models of transfinite provability logic. In Advances in Modal Logic, volume 9, pages 185-199. College Publications, 2012.

[25] D. Fernández-Duque and J. J. Joosten. Models of transfinite provability logic. To appear in Journal of Symbolic Logic. Preliminary version at http://personal.us.es/dfduque/glpmodels.pdf, 2012.

[26] D. Fernández-Duque and J. J. Joosten. Turing progressions and their wellorders. In How the world computes, Lecture Notes in Computer Science, pages 212-221. Springer, 2012.

[27] L. Esakia. Diagonal constructions, Löb's formula and Cantor's scattered spaces. In Studies in logic and semantics, pages 128-143. Metsniereba, Tbilisi, 1981. In Russian.

[28] S. Feferman, J.R. Dawson, S.C. Kleene, G.H. Moore, R.M. Solovay, and J. van Heijenoort, editors. Kurt Gödel Collected Works, Volume 1: Publications 1929-1936. Oxford Univeristy Press, 1996.

[29] D. Fernández-Duque. The polytopologies of transfinite provability logic. Preprint arXiv:1207.6595v2 [math.LO], 2012.

[30] K. Gödel. Eine Interpretation des intuitionistischen Aussagenkalkuls. Ergebnisse Math. Kolloq., 4:39-40, 1933. English translation in [28, pages 301-303.

[31] L. Harrington and S. Shelah. Some exact equiconsistency results in set theory. Notre Dame Journal of Formal Logic, 26:178-188, 1985.

[32] T. Icard and J. Joosten. Provability and interpretability logics with restricted realizations. Notre Dame Journal of Formal Logic, 53(2):133-154, 2012.

[33] T.F. Icard, III. A topological study of the closed fragment of GLP. Journal of Logic and Computation, 21(4):683-696, 2011.

[34] K.N. Ignatiev. On strong provability predicates and the associated modal logics. The Journal of Symbolic Logic, 58:249-290, 1993.

[35] G.K. Japaridze. The modal logical means of investigation of provability. Thesis in Philosophy, in Russian, Moscow, 1986.

[36] T. Jech. Set Theory. The Third Millenium Edition. Springer, 2002.

[37] A. Kanamori. The Higher Infinite. Second edition. Springer, 2009.

[38] M.H. Löb. Solution of a problem of Leon Henkin. The Journal of Symbolic Logic, 20:115-118, 1955.

[39] A. Macintyre and H. Simmons. Gödel's diagonalization technique and related properties of theories. Colloq. Math., 28:165-180, 1973.

[40] R. Magari. The diagonalizable algebras (the algebraization of the theories which express Theor.:II). Bollettino della Unione Matematica Italiana, Serie 4, 12, 1975. Suppl. fasc. 3, 117-125. 
[41] M. Magidor. Reflecting stationary sets. The Journal of Symbolic Logic, 47:755-771, 1982.

[42] J. C. C. McKinsey and A. Tarski. The algebra of topology. Annals of Mathematics, 45:141-191, 1944.

[43] A. Mekler and S. Shehlah. The consistency strength of "every stationary set reflects". Israel Journal of Mathematics, 67(3):353-366, 1989.

[44] H. Simmons. Topological aspects of suitable theories. Proc. Edinburgh Math. Soc. (2), 19:383-391, 1975.

[45] C. Smoryński. Self-Reference and Modal Logic. Springer-Verlag, Berlin, 1985.

[46] R.M. Solovay. Provability interpretations of modal logic. Israel Journal of Mathematics, 28:33-71, 1976.

[47] C. Steinsvold. Topological models of belief logics. PhD thesis, New York, 2007.

[48] A. Visser. The provability logics of recursively enumerable theories extending Peano Arithmetic at arbitrary theories extending Peano Arithmetic. Journal of Philosophic Logic, 13:97-113, 1984. 